\title{
Pulsar-Driven Jets in Supernovae, Gamma-Ray Bursts, and the Universe
}

\author{
John Middleditch \\ Los Alamos National Laboratory, CCS-3, MS B256, Los Alamos, NM 87544, USA \\ Correspondence should be addressed to John Middleditch, jon@lanl.gov \\ Received 18 April 2012; Revised 2 August 2012; Accepted 17 September 2012 \\ Academic Editor: Alberto J. Castro-Tirado \\ Copyright () 2012 John Middleditch. This is an open access article distributed under the Creative Commons Attribution License, \\ which permits unrestricted use, distribution, and reproduction in any medium, provided the original work is properly cited.
}

The bipolarity of Supernova 1987A can be understood through its very early light curve from the CTIO $0.4 \mathrm{~m}$ telescope and IUE FES and following speckle observations of the "Mystery Spot". These indicate a beam/jet of light/particles, with initial collimation factors $>10^{4}$ and velocities $>0.95 \mathrm{c}$, involving up to $10^{-5} \mathrm{M}_{\odot}$ interacting with circumstellar material. These can be produced by a model of pulsar emission from polarization currents induced/(modulated faster than $c$ ) beyond the pulsar light cylinder by the periodic electromagnetic field (supraluminally induced polarization currents (SLIP)). SLIP accounts for the disruption of supernova progenitors and their anomalous dimming at cosmological distances, jets from Sco X-1 and SS 433, the lack/presence of pulsations from the high-/low-luminosity low-mass X-ray binaries, and long/short gamma-ray bursts, and it predicts that their afterglows are the pulsed optical-/near-infrared emission associated with these pulsars. SLIP may also account for the $\mathrm{TeV}^{+} / \mathrm{e}^{-}$ results from PAMELA and ATIC, the WMAP "Haze"/Fermi "Bubbles," and the $r$-process. SLIP jets from SNe of the first stars may allow galaxies to form without dark matter and explain the peculiar nongravitational motions between pairs of distant galaxies observed by GALEX.

\section{Introduction}

Supernova 1987A has provided astronomers with a wealth of data, some of which have not even now, a quarter century after the event, been satisfactorily accounted for by any model. One of the most remarkable features in the early study of SN 1987A was the "Mystery Spot," with a thermal energy of $10^{49} \mathrm{erg}$, observed 50 days after the core-collapse event $[1-3]$ and separated from the SN photosphere "proper" by $0.060 \pm 0.008$ arcs at day 38 (Figure 1), with about 3\% of this energy eventually radiated in the optical band. The possibility that the enormous energy implied for the Mystery Spot might somehow link it to gamma-ray bursts (GRBs) attracted little serious consideration at the time, or even since, beyond a very astute few [4-6]. The Mystery Spot was also observed at separations of $0.045 \pm 0.008$ arcs on day 30 and $0.074 \pm 0.008$ arc s on day 50 but always at an angle of $194^{\circ}$, consistent with the southern (and approaching) extension of the bipolarity [7]. The Mystery Spot offsets from SN 1987A imply a minimum projected separation of $\sim 10$ light-days $(\ell t-d)$.
There is also a wealth of photometric and spectroscopic data from even earlier stages of SN 1987A, in particular photometry data from the Cerro Tololo Inter-American Observatory (CTIO) $0.4 \mathrm{~m}$ telescope [8] and the fine error sensor (FES) of the International Ultraviolet Explorer (IUE [9]), and spectroscopic data from [10, 11], among others. Short timescale structure $(\leq 1 \mathrm{~d})$ in this data, following finite delays ( $\sim 10 \mathrm{~d})$ after SN 1987A core collapse, implies at least one beam of light and jet particles which were initially highly collimated $\left(>10^{4}\right)$, interacting with circumstellar material.

GRBs, particularly long soft GRBs ( $\ell$ GRBs), appear to be the most luminous events in the Universe, occurring at the $\mathrm{SN}$ rate of one per second (assuming a collimation factor near $10^{5}$ ), yet we still know very little about them (see, e.g., [12] and references therein). A few $\ell$ GRBs have been found to be associated with $\mathrm{SNe}$ [13-15]. Others however, mostly those with slightly harder spectra and lasting only $\sim 1$ second (sGRBs), usually produce no lingering evidence at all, and only rarely the "afterglows" observed for a large proportion of $\ell$ GRBs, sometimes extending down to radio wavelengths. A large number of models have 
been put forth to explain GRBs, including neutron starneutron star mergers for sGRBs, and exotic objects such as "collapsars" [16] for $\ell$ GRBs. Part of the physical motivation for these, and the nearly universal use of their usually severe degrees of collimation, is the enormous energy of up to $10^{54} \mathrm{erg}$ implied for an isotropic source. Indeed, the data from SN 1987A presented herein, when interpreted through the model discussed in detail within this work, support a beam/jet collimation factor $>10^{4}$ in producing its early light curve by interaction with more or less stationary circumstellar material. In addition, the model predicts that GRB afterglows, and by extension of the principles of supraluminal excitations, even GRBs themselves, may dim only as distance $^{-1}$ (see Section 4), and thus easily removes the requirement for such a high energy.

Specifically, this work argues that polarization currents induced beyond the light cylinders of, and by the rotating magnetic fields from, newly formed pulsars embedded within their stellar remnants $[17,18]$ can account for the bipolarity of SN 1987A [19-21]. This model of emission from supraluminally induced polarization currents (SLIPs) provides a mechanism for generating a pulsed beam, rotating on the surface of a cone whose half angle (and angle from the pulsar axis of rotation) is given by

$$
\theta_{V}=\arcsin \frac{c}{v}
$$

for astronomical distances. Here, $c$ is the speed of light and $v>c$ is the speed at which the polarization currents are updated, that is, $v=\omega R$, where $\omega$ is the pulsar rotation frequency in radians $\mathrm{s}^{-1}, R>R_{\mathrm{LC}}$ is the distance of the polarization current from the pulsar projected onto the rotational equatorial plane, and $R_{\mathrm{LC}}$ is the light cylinder radius $\left(\omega R_{\mathrm{LC}}=c\right)$.

Emission from circularly supraluminal polarization currents is initially focused as a single point, which moves around the equator of the light cylinder at the angular pulsar rate, but which eventually travels outward on the two cones defined by (1). When the emission is observed for the conal directions, the pulse profile will consist of only a single sharp peak and will have an intensity that decays only with the first power of distance $[22,23]$ (see Section 4$)^{1}$.

This emission mechanism, and its unusual distance law, will operate at any radius beyond the light cylinder where sufficient plasma exists. In principle, this would render pulsations nearly always visible from neutron stars surrounded by plasma. However, pulsations are almost never seen from neutron stars which are born inside of SNe with $\mathrm{M}_{\odot}$ 's of material yet to eject, and those in the nontransient (and more luminous) low-mass X-ray binaries (LMXBs). What happens is that emissions from smaller annuli must push out through the densest material of the stellar core or circumpulsar accretia, while emissions from larger annuli, although encountering more material on the way to the equatorial light cylinder, also bypass much of the core material while traveling toward the poles. This results in a greater beam concentration near the rotational poles, and thus, the solid angle for which pulsations are observable from $\mathrm{SNe}$ and LMXBs is tiny and is almost never expected to be

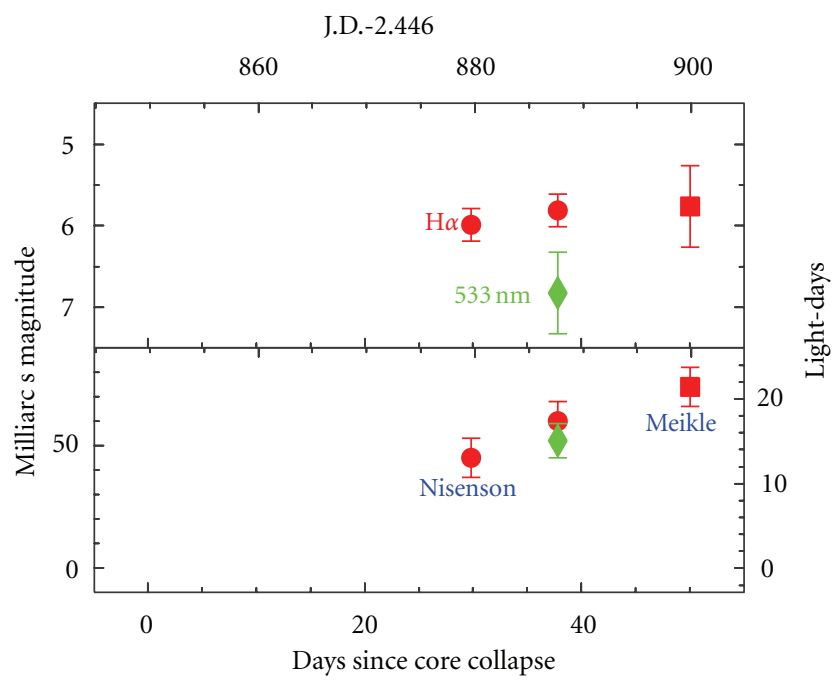

Figure 1: Measurements of displacement (lower) and observed magnitude (upper) of the "Mystery Spot" from SN 1987A, at $\mathrm{H} \alpha$ and $533 \mathrm{~nm}$, versus time, from [1-3].

seen (an SN Ic, however, is worth observing — see Section 5). In the case of $\mathrm{SNe}$, the beam accelerates (and transmutes) matter from the stellar interior, which includes $\mathrm{H}, \mathrm{He}, \mathrm{C}, \mathrm{O}$, and heavier elements for massive solitary progenitor stars.

In the rest of this paper, Section 2 includes a quantitative discussion of the SN 1987A early luminosity history and motivations for why a later quasisteady less collimated, as well as a prompt highly collimated injection events are needed. Then Section 3 estimates the kinematics and the working geometry for the 1987A beam/jet and Mystery Spot, and we explore the implications of these on the process which gave rise to SN 1987A and the observations during the following years. Section 4 relates the SN 1987A beam/jet process to GRBs, germinates the idea that their afterglows are highly pulsed, ${ }^{2}$ and introduces the possibility that $1987 \mathrm{~A}$ itself was the result of a merger of two electron degenerate stellar cores within a common envelope-double degenerate, or DD—while Section 5 relates the process to Type Ia/c SNe. Section 6, including Sections 6.1-6.5, extends the discussion of the implications of the DD process to the formation and luminosities of Type Ib and II SNe, the relation of plasma available to the SLIP process and transient signals (Section 6.2), Sco X-1 and other low-mass X-ray binaries (LMXBs, Section 6.3), historic SNe, pulse profiles, and SNR bipolarity (Section 6.5) . Section 6.6 discusses how SLIP explains why PSR J0537 is predominantly an X-ray but not an optical pulsar. Section 6.7 discusses the high kinetic energy of the SN 1987A jet(s) and its possible relation to the positron excess, WMAP "Haze", Fermi "Bubbles", and $r$-process, followed by a discussion of the unique SS 433 jets in Section 6.8, pulsar-driven jets in the early Universe in Section 6.9, and pulsar "recycling" in Section 6.10. Section 7 concludes, and a short SLIP primer follows in the appendix. 


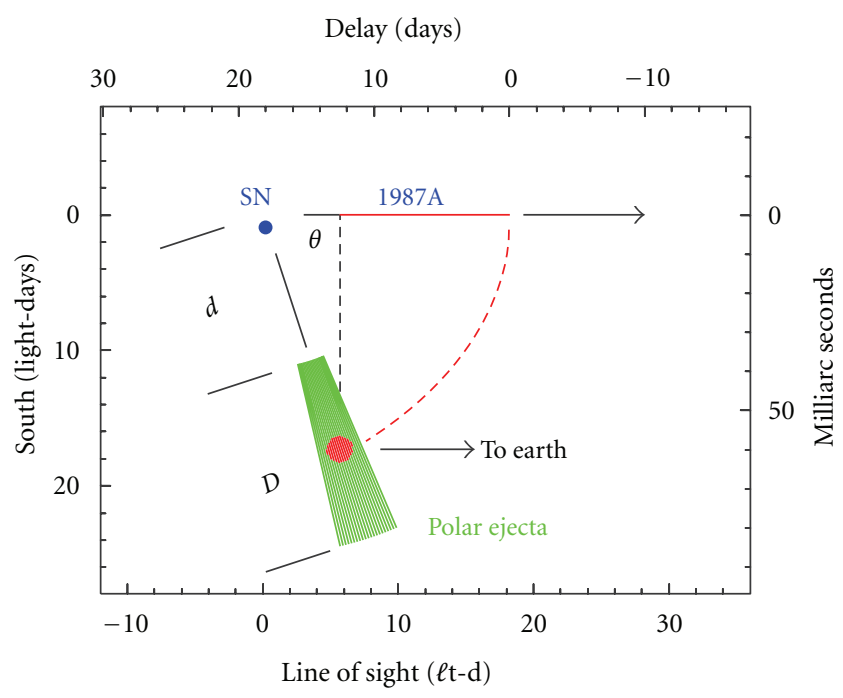

Figure 2: The geometry of the "Mystery Spot," (red dot) polar ejecta, associated beam/jet, and direct line of sight from SN 1987A to the Earth.

\section{The Early Luminosity History of SN 1987A}

Table 1 gives an event history for SN 1987A and its progenitor system. An approximate geometry for SN 1987A and a Mystery Spot located within circumstellar material (in this case, polar ejecta) are given in Figure 2, while the early luminosity histories of SN 1987A from CTIO and the fine error sensor (FES) of the IUE, which we can use to refine the parameters that this figure postulates as reflecting reality, are both plotted in Figure 3. ${ }^{3}$

Following the drop from the initial flash, the luminosity rises again to a maximum ("A" in Figures 3 and 4(a)) of magnitude 4.35 at day 3.0 , roughly corresponding to $2.7 \times$ $10^{41} \mathrm{erg} \mathrm{s}^{-1}$ and interpretable as a luminous jet emerging from cooler outer layers which initially shrouded it. This declines to magnitude 4.48 around day 7.0 ("B", Figure 4(b)), interpretable as free-free cooling or the loss of the ability to cool, as the jet becomes more diffuse. The next observable event should be the scattering/reprocessing of the initial UV flash in the polar ejecta at day $\sim 8$, and indeed, " $\mathrm{C}$ " (Figure 4(c)) shows $\sim 2 \times 10^{39} \mathrm{erg} \mathrm{s}^{-1}$ in the optical for a day at day 7.8, and a decline consistent with the flash after that, indicating that no significant smearing over time had occurred in this interaction. The 8-day delay to, and the rapid decline of, this first event implies a collimation factor $>10^{4}$ for this part of the UV flash. This can be seen if we let $\delta$ represent the radius of the illuminated area in the polar ejecta, $d$ as in Figure 2 is the distance from the SN to this area, the collimation factor $\Upsilon$ for the beam can be expressed as

$$
\Upsilon=2 \pi\left(\frac{d}{\delta}\right)^{2}
$$

or

$$
\Upsilon=10^{4}\left(\frac{d}{10 \ell \mathrm{t}-\mathrm{d}}\right)^{2}\left(\frac{\delta}{0.25 \ell \mathrm{t}-\mathrm{d}}\right)^{-2}
$$

TABLE 1: SN 1987A Event Log.

\begin{tabular}{lc}
\hline $\begin{array}{l}\text { Time } \\
t\end{array}$ & Event \\
\hline$-20,000$ years & Rings formed \\
$\sim(-2,000$ ? $)$ years & Polar, or near-polar ejection \\
$0(=\mathrm{UT} 1987$, Feb. 23.316) & Core-collapse of SN 1987A \\
$0<t<2$ days & UV flash from SN 1987A \\
$2<t<4$ days & Emergence of luminous jet \\
$4<t<7$ days & Cooling/spreading of jet \\
7.8 days & UV flash hits polar ejecta \\
8.26 days & Jet impacts polar ejecta (PE) \\
19.8 days & Pulsations clear through PE \\
20.8 days & Jet particles clear through PE \\
30 days & "Mystery spot" at 45 mas \\
38 days & "Mystery spot" at 60 mas \\
50 days & "Mystery spot" at 74 mas \\
\hline
\end{tabular}

A linear ramp in luminosity, visible by day 9.8 (" $\mathrm{D}$ " in Figures 3 and 5(a)), indicates particles from the jet penetrating into the polar ejecta, with the fastest traveling at $>0.8 \mathrm{c}$, and because of the sudden rise, a particle collimation factor $>104$. Back extrapolation of the three CTIO points just after day 8 intersects the day 7 minimum near day 8.26 , which would indicate that particles exist in this jet with velocities up to $0.95 \mathrm{c}$, and even higher if the true minimum flux is lower than the points at magnitude $4.48\left(1.6 \times 10^{41} \mathrm{erg} \mathrm{s}^{-1}\right)$ near day 7.

The ramp continues until after day 20 , when a decrement of $\sim 5 \times 10^{39} \mathrm{erg} \mathrm{s}^{-1}$ appears in both data sets just after day 20 ("F" in Figures 3 and 5(c)). The CTIO point just before the decrement can be used as a rough upper limit for the Mystery Spot luminosity and corresponds to an excess above the minimum (near day 7.0) of $5 \times 10^{40} \mathrm{erg} \mathrm{s}^{-1}$, or magnitude 5.8, the same as that observed in $\mathrm{H} \alpha$ for the Mystery Spot at days 30,38 , and 50 , about $23 \%$ of the total optical flux of $2.1 \times 10^{41} \mathrm{erg} \mathrm{s}^{-1}$ at that time.

This decrement is preceded by a spike ("E" in Figures 3 and 5(b), and Figure 6, day 19.8) of up to $10^{40} \mathrm{erg} \mathrm{s}^{-1}$ in the CTIO data, with the unusual colors of B, R, and I, in ascending order, very close to the $\mathrm{B}$ and I bands speculated for the $2.14 \mathrm{~ms}$ signature observed from 1987A [24, 25] with an $\mathrm{H} \alpha$ enhancement. Spectra taken by [25] around March 15 (the "Bochum event") [10] on March 14.820 (day 19.504), and [11] on UT 1987, March 15.08 (day 19.76), support these flux enhancements, including the $\mathrm{H} \alpha$ (and a Ca II triplet, $\lambda \lambda$ $849.8,854.2,866.2 \mathrm{~nm}$, as well). The timing of this event, one day prior to the decrement, suggests that it is due to a photon stream, stripped of its UV component by absorption (the CTIO U point at day 19.8 was low, consistent with this interpretation), scattering into other directions, including the line of sight to the Earth, by what might have been a clumpy end to the circumstellar material. Optical pulsations were not detected at this early epoch (R. N. Manchester, private communication 2007) because of the oblique view and the dimensions of the beam $(\sim 1 \ell \mathrm{t}-\mathrm{d})$. 


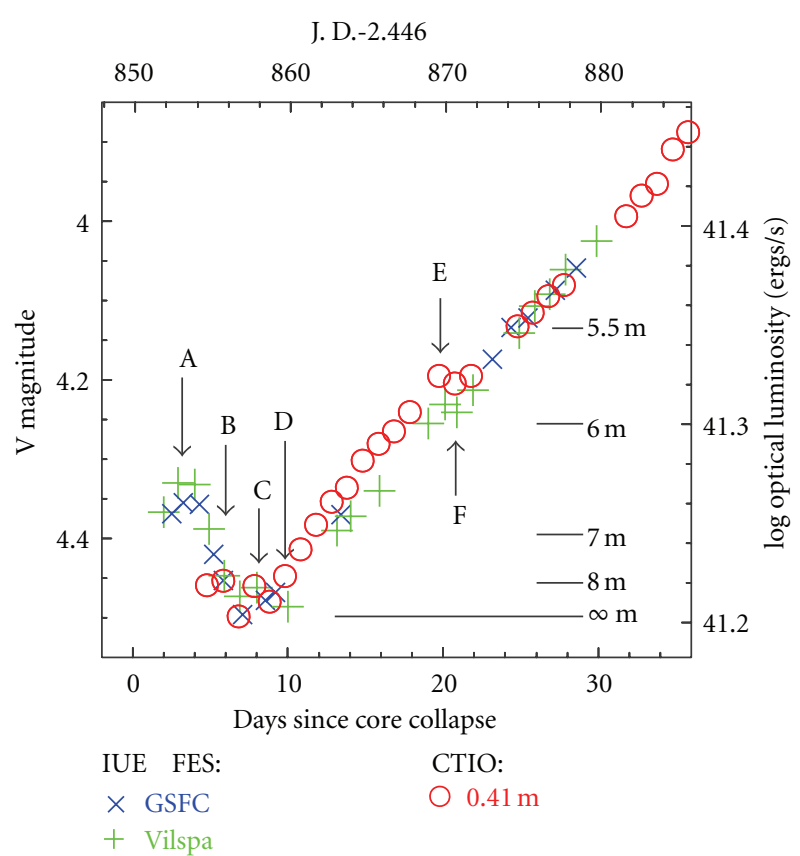

FIGURE 3: The very early luminosity history of SN 1987A as observed with the fine error sensor of IUE and the $0.41-\mathrm{m}$ at CTIO. Data points taken at Goddard Space Flight Center by Sonneborn \& Kirshner, and at the Villafranca Station in Madrid, are marked (see Section 2). The diameter of the circles representing the CTIO data is about three times the error. A V magnitude scale, for excess light above the minimum near day 7 , is given, within the frame, on the right.

For the geometry derived in Section 3 below, the one-day delay implies at least the same maximum jet velocity $(0.95 c)$, supporting this interpretation of a beam and jet penetrating extrastellar material and giving us a rough isotropic lowerlimit estimate of the strength of the pulsations. Spectra taken just before day 5, showing an enhancement for wavelengths below $5000 \AA$, explain the discrepancy between the CTIO and FES points at that time (Figure 3 ).

In spite of the coincidence between the end magnitude of the linear ramp and that of the Mystery Spot, the two are probably not of the same effect, as the offset of the Mystery Spot from SN 1987A was only 0.045 arc s even 10 days later at day 30 (Figure 7), a location barely beyond where the ramp began, as is shown below, and there is no sign of elongation toward SN 1987A proper in Figure 1 of [3] or Figure 2 of [2]. The Mystery Spot may develop as a plume within the polar ejecta, pushed by a less collimated $0.5 c$ pulsar wind, perhaps not unlike that observed from the Crab pulsar [26], many days after the passage of the initial, very fast, very collimated component of the jet. A beam only $1 \mathrm{lt}$-d across at $\sim 10 \mathrm{lt}-\mathrm{d}$ translates via (1) into plasma at $\sim 20 R_{\mathrm{LC}}$.

It is unlikely that the early light curve is due to shallow penetration of a precessing jet into a varying entry point of the polar ejecta because the high density required to limit jet penetration comes with a higher opacity which would make the linear ramp hard to produce in this, the inner boundary of the approaching axial feature. The requirement for an $0.5 \mathrm{c}$ mean motion of the Mystery Spot between days 30 and 38, slowing to $0.35 \mathrm{c}$ between days 38 and 50 (ostensibly due to sweptup matter), would also be difficult to fulfill in these circumstances. On the other hand, the polar ejecta density cannot be so low as to allow more than $1 \mathrm{lt}$-d penetration by the enhanced UV flash, or the drop in luminosity from day 7.8 to day 8.8 would not be as big. If the jet penetration is deep, precession and/or changes in the plasma density beyond the pulsar light cylinder (1) may make its initial track, within the polar ejecta, a helical cone, and this may assist the $\sim 0.5 c$ wind in the creation of the plume which forms the Mystery Spot within three weeks of the initial jet penetration.

We will assume that the optical flux from the interaction between jet particles and the polar ejecta will not be significantly occulted in the ejecta itself in the direction to the Earth, otherwise again, the linear ramp would be difficult to produce. As we will find below that the axis of the $\mathrm{SN}$ $1987 \mathrm{~A}$ bipolarity is $\sim 30^{\circ}$ from the normal to the ring planes, the reason is remaining a mystery even today, this is not necessarily a given. Proceeding nevertheless is as follows. By scaling homologously inward a factor of 10 from the equatorial ring density of $10^{4} \mathrm{~cm}^{-3}$, we arrive at a polar ejecta density estimate of $\sim 10^{7} \mathrm{~cm}^{-3}$ - sufficient to stop the UV flash from penetrating $>1 \mathrm{\ell t}-\mathrm{d}$.

Assuming a polar ejecta depth, $D$, of $\sim 10 \ell \mathrm{t}-\mathrm{d}$, or $2.6 \times$ $10^{16} \mathrm{~cm}$, gives a total column of $2.6 \times 10^{23} \mathrm{~cm}^{-2}$, sufficient to hopelessly disperse any pulsed radio signature, though enough to warrant some concerns about continued particle penetration. However, only a fraction of the protons in the jet will scatter through the entire depth of the polar ejecta (the slight concave downward departure from linearity most apparent in the CTIO data, between days 9 and 20, may reflect this loss, and/or the density in the polar ejecta may decrease with distance). In addition, we will find that the angle, $\theta$, from our line of sight to the SN 1987A beam/jet will be large in the self-consistent solution, justifying our assumption of visibility for the luminous column within the polar ejecta between days 9 and 20 .

\section{The Geometry and Kinematics of the Beam/Jet}

Using the constraints shown in Figures 1 and 3, we can solve for the three geometric variables, $d, D$, and $\theta$ (diagrammed in Figure 2), and the maximum velocity of the particles in the jet, $\beta$. First, the UV flash hits the beginning of the polar ejecta at day 7.8 as follows:

$$
d(1-\cos \theta)=c t_{0}==7.8 \ell \mathrm{t}-\mathrm{d}
$$

where $d$ is the distance to the beginning of the polar ejecta, $\theta$ is the angle from the beam/jet/polar ejecta direction to the line of sight to the Earth, and $c$ is the speed of light.

From Figure 3, we also have the jet particles well into the polar ejecta by day 9.8. Extrapolating the three CTIO points 


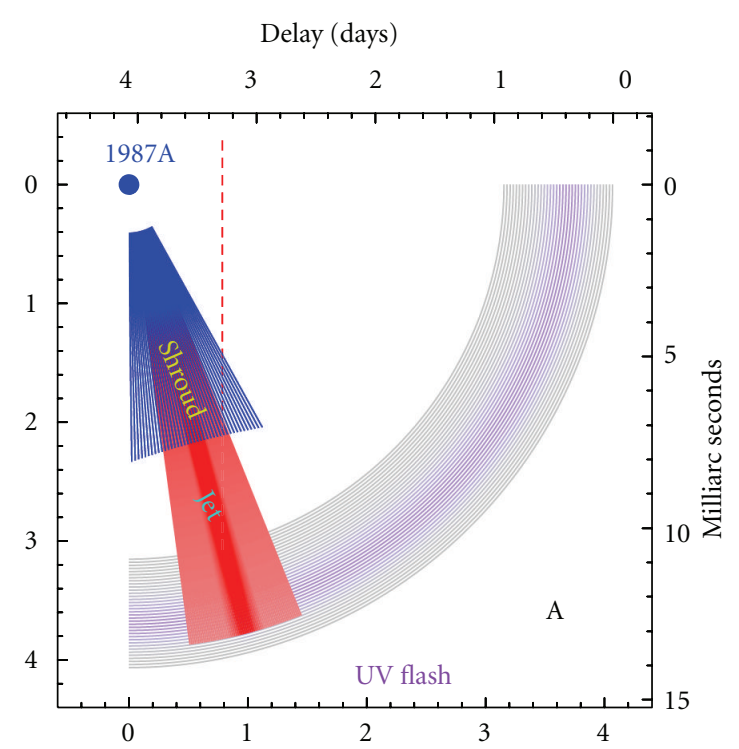

(a)

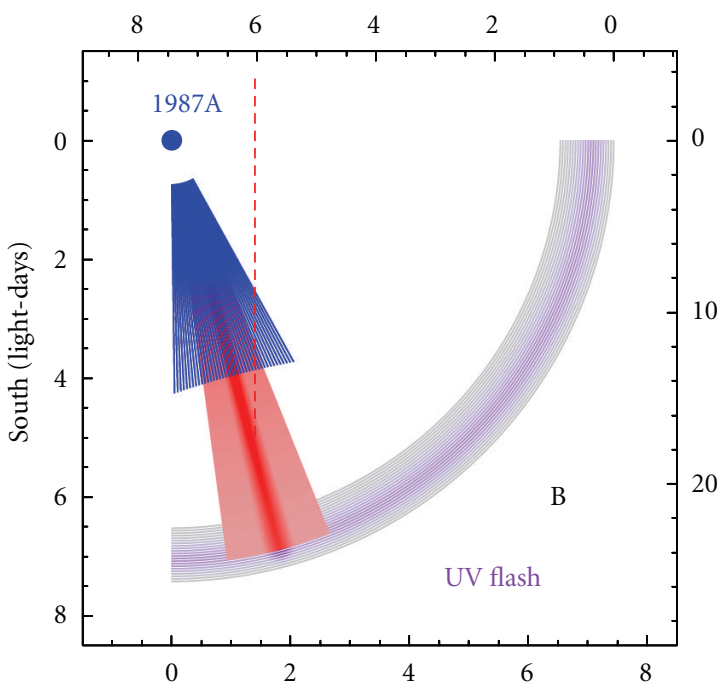

(b)

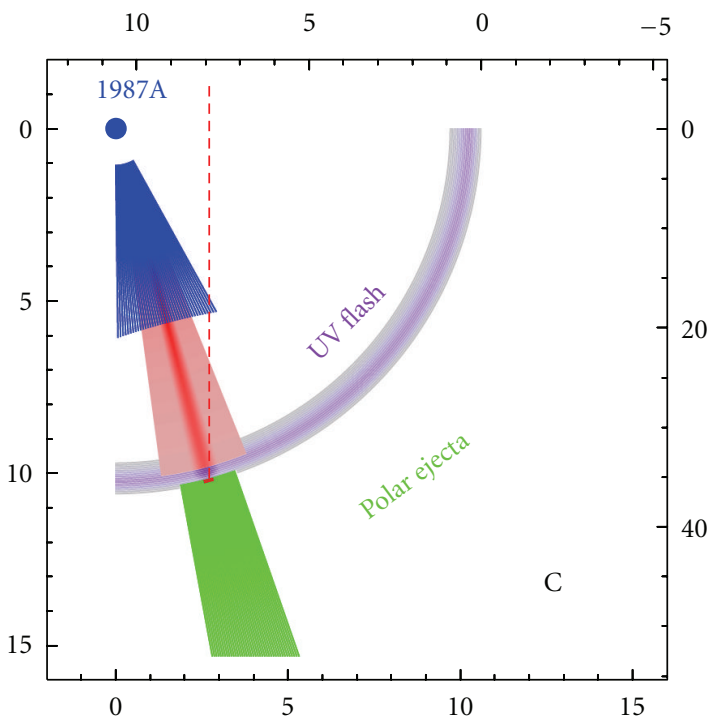

(c)

FIGURE 4: The geometry of the 1987A-glowing beam/jet, initially opaque shroud, and UV flash (which may have an enhanced beam of its own in the jet direction here $75^{\circ}$, down and to the right). The maximum velocity of the jet/shroud is $0.95 / 0.55 \mathrm{c}$. The dashed line to the upper scale flags the center of the emerging jet at day $3.3((\mathrm{a})-\mathrm{A})$ and day $6((\mathrm{~b})-\mathrm{B})$, and the UV flash hitting the polar ejecta at day 7.8 $((\mathrm{c})-\mathrm{C})$.

(just after day 8) backward per above, we have the fastest beam particles hitting the polar ejecta at day 8.26 as follows:

$$
d\left(\frac{1}{\beta}-\cos \theta\right)=c t_{1}==8.26 \ell \mathrm{t}-\mathrm{d}
$$

Next, we have the projected offset of $0.060 \mathrm{arcs}$ for the Mystery Spot measured at day 29.8 by [2] and refined by [3]. This is more difficult to pin down relative to its position radially through the polar ejecta, so we assume it is some fraction, $\alpha$, of the way through the polar ejecta depth, $D$, and derive a self-consistent solution as follows:

$$
(d+\alpha D) \sin \theta=c t_{2}==17.3 \ell \mathrm{t}-\mathrm{d},
$$

using $50 \mathrm{kpc}$ for the distance to SN 1987A. Finally, we have the decrement in the light curve at day 20, shown in Figure 3, which we will interpret as the fastest "substantial" bunch of particles in the jet breaking through the end of the polar ejecta as follows:

$$
(d+D)\left(\frac{1}{\beta}-\cos \theta\right)=c t_{3}==20 \ell \mathrm{t}-\mathrm{d} .
$$




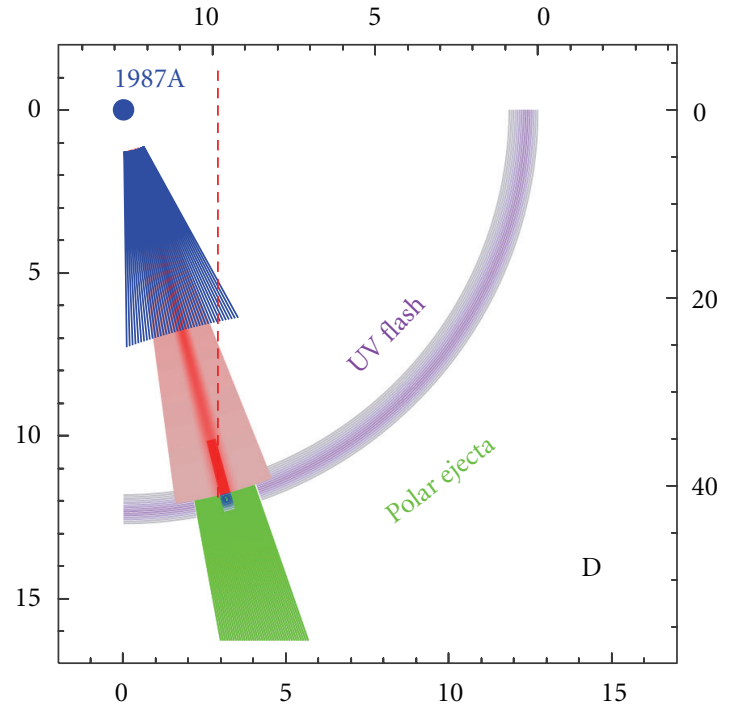

(a)

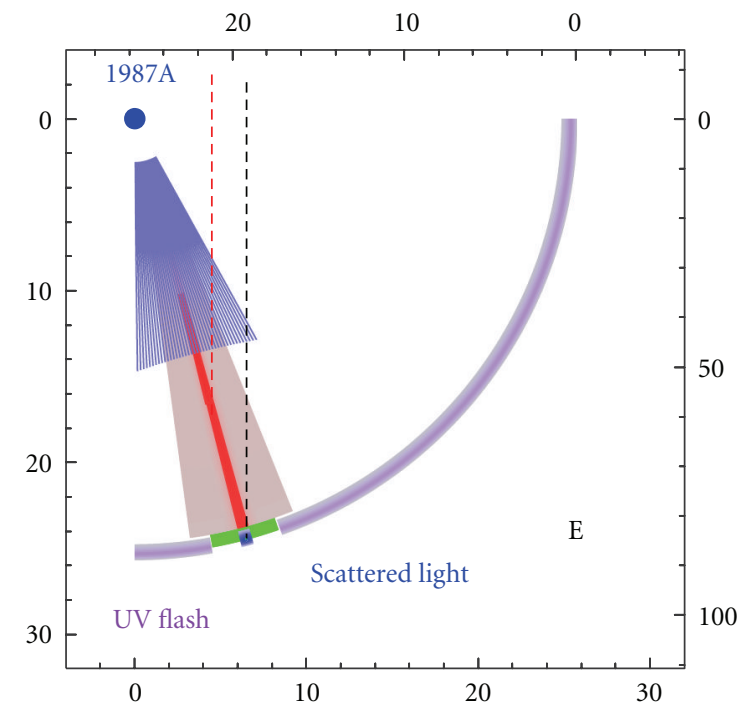

(b)

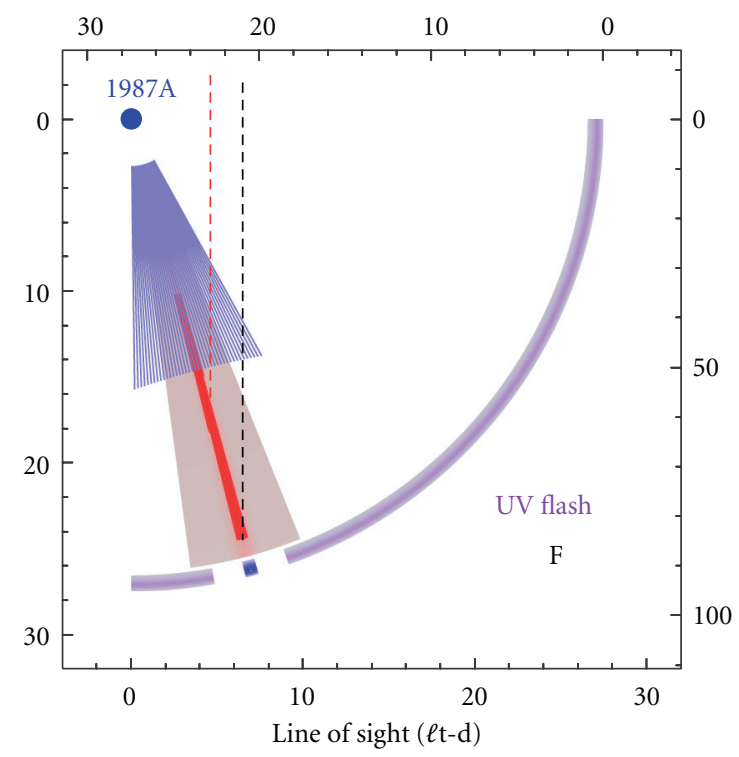

(c)

FIgURE 5: ( (a)-D). The intense center $\left(\sim 1^{\circ}\right)$ of the jet begins to produce light as it penetrates into the polar ejecta, producing the jump in luminosity at day 9.8. ((b)_E) Particles in the jet continue to impact the polar ejecta (mostly hidden), extending the ramp in luminosity visible in Figure 3 near day 20 (left dashed line to the top scale). Right dashed line: light from the filtered UV flash scatters in clumpy polar ejecta near its outer boundary. ((c)—F) The fastest jet particles have cleared the end of the polar ejecta.

The solution to (4)-(7) gives a constant ratio for $D$ to $d$, independent of $\alpha$ as follows:

$$
d=\frac{D t_{1}}{\left(t_{3}-t_{1}\right)}, \quad \text { or } \quad(d+D)=\frac{d t_{3}}{t_{1}}
$$

while $\theta$ is given by

$$
\theta=2 \tan ^{-1}\left\{\frac{t_{0}}{t_{2}}\left(\alpha\left(\frac{t_{3}}{t_{1}}-1\right)+1\right)\right\}
$$

The parameters, $d, D+d$, and $\theta$, are plotted against $\beta$ in Figure 8 for $0.3 \leq \alpha \leq 0.7$, along with the maximum $d$ and minimum $D+d$ implied by the three measurements of the Mystery Spot angular separation at days 30, 38, and 50. Figure 8 shows that the polar ejecta, at the very least, must start by $14 \mathrm{lt}-\mathrm{d}$ or closer and extend to $22 \mathrm{lt}-\mathrm{d}$ or farther, consistent with our early $10 \mathrm{lt}$-d estimates for $d$ and $D$ as is the high value of $\theta\left(65^{\circ}<\theta<85^{\circ}\right)$, which also means that the axis of bipolarity is $\sim 30^{\circ}$ from the normal to the ring planes [27].

Given the similar magnitudes of the early light curve and the Mystery Spot (and indeed, the two are just phases of the same jet phenomenon; the first being more penetrating and the second more energy injecting), the energetics of the 


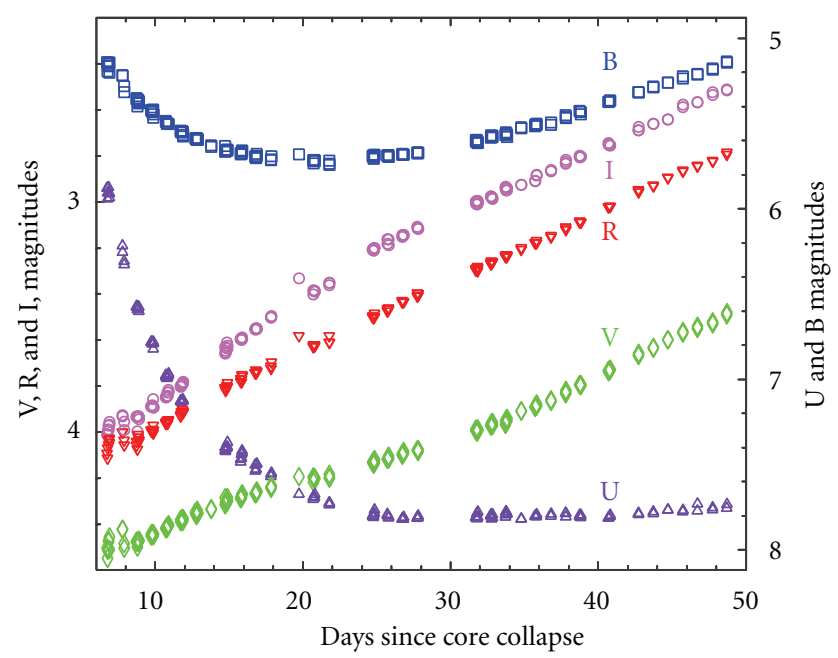

Figure 6: The U, B, V, R, and I points from the CTIO 0.41-m from days 6 to 50 (see Section 2).

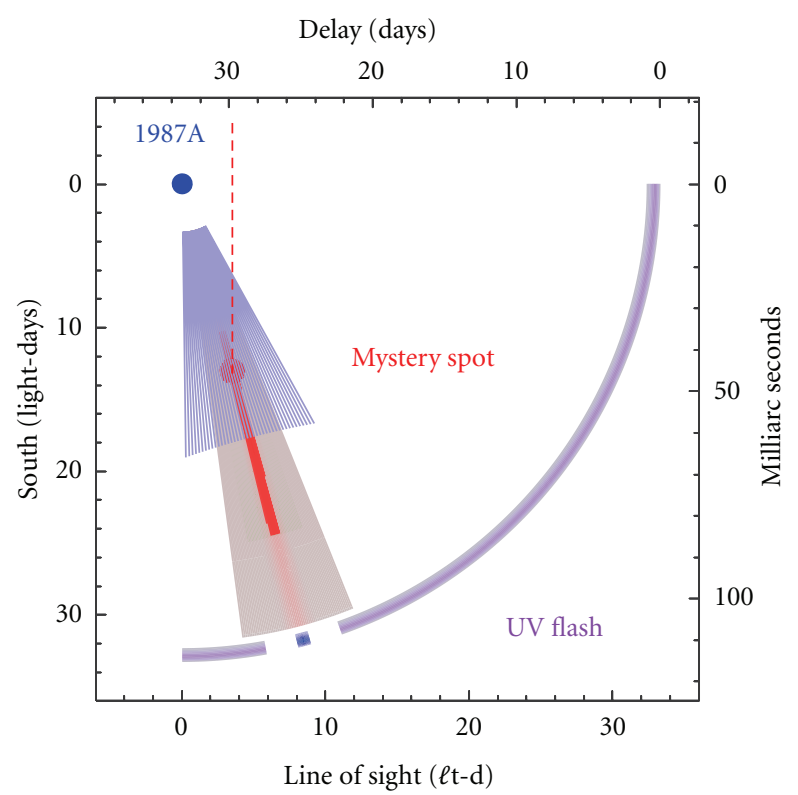

Figure 7: The relation of the Mystery Spot, near day 30, to a jet, a thinning shroud, and a UV Flash, when its projected offset from SN 1987A was 0.045 arc s.

jet producing the early light curve and these are the same as that posited for the Mystery Spot in [1], except that the early light-curve phase is shorter. For an interval of $10^{6} \mathrm{~s}$, at $5 \times 10^{40} \mathrm{erg} \mathrm{s}^{-1}$, the optical output, from reprocessing of X-rays resulting from the jet particles scattering with electrons, which then add to it through free-free or later synchrotron radiation, is $5 \times 10^{46} \mathrm{erg}$. Since only a fraction of the particles scatter in the polar ejecta, the overall efficiency, in the conversion of kinetic energy into optical luminosity, could still be as low as Meikle et al.'s estimated 0.001, which gives $5 \times 10^{49}$ erg of kinetic energy in the initial jet. For $0.9 \mathrm{c}$ protons, each with $0.002 \mathrm{erg}$ of kinetic energy, this would mean $2.5 \times 10^{52}$ protons or $2 \times 10^{-5} \mathrm{M}_{\odot}$ initially for each

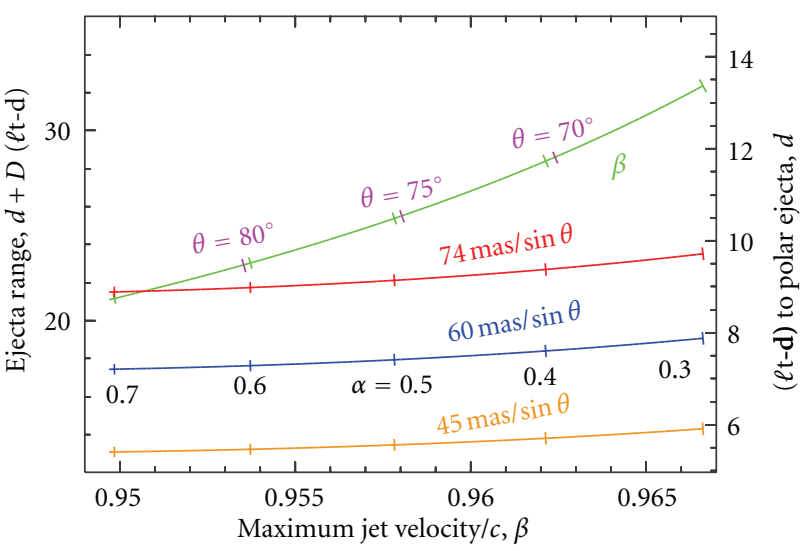

Figure 8: The solution values for (4)-(7). Horizontal: the maximum jet velocity, $\beta$. Left vertical: the maximum range of the polar ejecta. Right vertical axis: the distance from the pulsar to the beginning of the polar ejecta. The line with the steepest slope converts $\beta$ (bottom) to $D+d$ (left) or $d$ (right), and three values for $\theta$ are marked. The three other lines with moderate slopes constrain the minimum of $D+d$ (right end of 74 mas curve) and the maximum of $d$ (left end of the 45 mas curve and also read on the left vertical axis) from offset measurements of the Mystery Spot, which is assumed to be a jet-driven plume within the polar ejecta. Five values of $\alpha$, from 0.3 to 0.7 , are tic-marked on the four curves.

jet. Without the now visible counterjet, the "kick" velocity to the neutron star would only be $10 \mathrm{~km} \mathrm{~s}^{-1}$, thus such jets are unlikely to produce the $100 \mathrm{~s}$ of $\mathrm{km} \mathrm{s}^{-1}$ velocities seen in a few other pulsars.

For a pulsar with an initial spin rate of $500 \mathrm{~Hz}$, this short phase alone would result in a drop of $10 \mathrm{~Hz}$, corresponding to a mean spindown rate of $10^{-5} \mathrm{~Hz} \mathrm{~s}^{-1}$, assuming a neutronstar moment of inertia of $5 \times 10^{44} \mathrm{~g}-\mathrm{cm}^{2}$. This may still be an underestimate, as accelerating a square $\ell \mathrm{t}-\mathrm{d}$ of the polar ejecta column, which amounts to $\sim 0.002 \mathrm{M}_{\odot}$ to $\sim 0.3 \mathrm{c}$, requires $1.6 \times 10^{50} \mathrm{erg}$ of kinetic energy, which amounts to $6.6 \%$ of the $2.5 \times 10^{51} \mathrm{erg}$ of rotational energy of a $500 \mathrm{~Hz}$ pulsar for each jet, or $\sim 66 \mathrm{~Hz}$ of frequency drop from $500 \mathrm{~Hz}$, still assuming $100 \%$ conversion of jet kinetic energy into Mystery Spot kinetic energy, unless the plume has a smaller cross section than $1 \mathrm{lt}-\mathrm{d}^{2}$ and/or the polar ejecta is less dense, on average, than $10^{7} \mathrm{~cm}^{-3}$. Since these numbers account only for the near polar SLIP beam(s), and not to the more equatorial beams, they must be considered to be lower limits. Observations of initial pulsar spindowns (see Section 4) would help greatly in resolving this uncertainty. Spinup from accretion may temper the spindown somewhat [28], but gravitational radiation reaction may counter it [29], though the high electromagnetic spindown will mask any effect of this latter on the pulsar braking index, $n$, where $(\partial f / \partial t) \propto-f^{n}$, and $n=5$ for pure gravitational radiation.

In either case, the rotational energy required is too large to be supplied by a strongly magnetized pulsar over the required timescale, unless these are born spinning faster than the moderate rates generally believed to be typical (e.g., the $\sim 12 \mathrm{~ms}$ initial period for the $16.1 \mathrm{~ms}$ PSR J05376910 [30] — see Section 6.6). There was certainly no evidence 


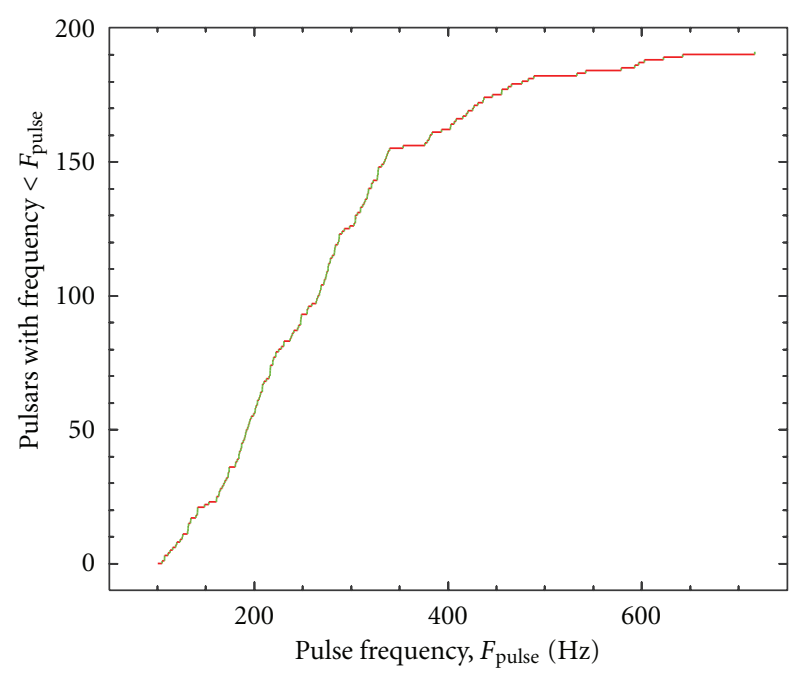

FIgURE 9: The distribution of pulsars from the ATNF pulsar database with spin rates greater than $100 \mathrm{~Hz}$ (http://www.atnf .csiro.au; [49]).

for a strongly magnetized pulsar within SN 1987A in its first few years (e.g., [31-33]), and most importantly, there is no evidence for such a pulsar in the last few years [34], whereas SN 1986J, at the same age, showed clear evidence of such a pulsar within it. ${ }^{4}$

However, there may be a weakly magnetized pulsar within SN 1987A [24, 35], and at the very least, this is supported by solid evidence for the at the very least this is supported by solid evidence for the formation of a neutron star $[36,37]$. A binary merger of two electron-degenerate stellar cores (DD-in isolation, these would be white dwarfs) has been proposed for SN 1987A [38], and the triple ring structure, particularly that of the outer rings, has recently been successfully calculated within this framework [39]. Other details of SN 1987A, including the mixing [40], the blue supergiant progenitor [41], the early polarization [4244 , and the possible $2.14 \mathrm{~ms}$ optical pulsations [24, 35], support this hypothesis.

The first clear evidence for DD-formed millisecond pulsars coincidentally came in the birth year of SN 1987A, with the discovery of the $3 \mathrm{~ms}$ pulsar, B1821-24 [45], in the noncore-collapsed globular cluster, M28. Subsequently, many more were found over the next 20 years in such clusters (e.g., 47 Tuc [46]), and attributing these to recycling through X-ray binaries has never really worked $[47,48]$, by a few orders of magnitude. ${ }^{5}$ Thus the DD process in SN 1987A, albeit within a common envelope, would likely have formed a rapidly spinning weakly magnetized pulsar.

Consequently, we also argue, as a corollary implication useful for understanding the SN process and its modern-day observation history, that the vast majority of core-collapse events are similar to SN 1987A, in that they are a result of the DD process, producing only weakly magnetized rapidly spinning ms pulsars, the notable exceptions being SNe 1986J, and 2006gy and 2007bi; these latter will be discussed further below. In spite of the rarity of recent $\mathrm{SNe}$ with solid evidence for a strongly magnetized neutron star remnant, many still currently hold that such a result is the more frequent result of stellar core-collapse events.

The above estimates for an early phase of drastic spindown are in line with the differences between the years-old spin period of the $2.14 \mathrm{~ms}(467 \mathrm{~Hz})$ signature for SN 1987A of $[24,35]$ as a Population I pulsar, ${ }^{6}$ and the Population II pulsars with spin periods just over $2 \mathrm{~ms}$, such as Ter 5 $\mathrm{Y}$ and $\mathrm{V}(2.05$ and $2.07 \mathrm{~ms}, 488 \mathrm{~Hz}$ and $482.5 \mathrm{~Hz})$ and 47 Tuc F $(2.10 \mathrm{~ms}, 476 \mathrm{~Hz})$. The pulsar, J0034-0534, has the next highest spin rate, at $532.7 \mathrm{~Hz}$, a gap of $44.5 \mathrm{~Hz}$. There are seven in the same frequency range below Ter $5 \mathrm{Y}$ (not counting SN 1987A), lending credibility to the commonality of $2 \mathrm{~ms}$ for the birth periods for pulsars produced by DD merger. ${ }^{7}$ This would also explain how ms pulsars could be recycled to spin rates faster than $500 \mathrm{~Hz}$ [50], without requiring any decay of the magnetic field. The occurrence of pulsars with various spin frequencies shows an increased density for frequencies just under $500 \mathrm{~Hz}$, consistent with this picture (see Figure 9). After a (significant) gap from 378 to $346 \mathrm{~Hz}$, where there is only PSR J0024-7204S at $353.3 \mathrm{~Hz}$, the pulsars slower than $346 \mathrm{~Hz}$, the steepest part of the curve, comprise the " $5 \mathrm{~ms}$ pileup," that is, those pulsars (in part) possibly are the offspring of defunct LMXBs (discussed further in Section 6.10). The pulsars between 378 and $500 \mathrm{~Hz}$ are not numerous enough to populate the pileup just by spinning down, without resorting to entities such as age and beaming differences.

Table 2 lists the anticipated emission and spindown phases of an SN/pulsar similar to 1987A. The epoch at which the common envelope transitions from bilobed to spheroidal is estimated at 2000 years prior to core collapse, at which time the spindown is dominated by SLIP losses to mass ejection/disruption of the progenitor star and thus is very high. The time range at which the losses transition from SLIP to gravitational radiation is estimated to be 2 to 4 years, after which GR losses are estimated to dominate for a century. At $-2 \times 10^{-10} \mathrm{~Hz} \mathrm{~s}^{-1}$, this amounts to a loss of $\sim 0.6 \mathrm{~Hz}$, which may be an overestimate, since the spindown may not sustain this rate over a century. Certainly the loss cannot amount to several dozens of $\mathrm{Hz}$, as the resulting tight grouping of pulsars with spin periods near $2 \mathrm{~ms}$ would not happen. When the pulsar is about a decade old, there is not enough plasma left (barring occasional clumps) to sustain optical pulsations. Thus, the epoch of optical pulsations for SN 1987A started near 4 years of age, which is relatively late, due to the large angle between the spin axis inferred from the bipolarity and the line of sight to the Earth, some $75^{\circ}$.

In this case, plasma initially available at many $R_{\mathrm{LC}}$ resulted in axially driven pulsations. Precession and nutation may have further embellished the axial pattern [35]. A strongly magnetized pulsar, because it will be spinning more slowly than its weakly magnetized counterparts, will have a larger $R_{\mathrm{LC}}$ and thus produce a much less collimated beam and jet than those of SN 1987A because plasma is available at far fewer $R_{\mathrm{LC}}$, but the larger progenitor counteracts this trend somewhat. As the 1987A circum neutron star density declined, polarization currents were restricted to fewer $R_{\mathrm{LC}}$, resulting in pulsed emission along a cone of finite polar 
TABLE 2: $15 \mathrm{M}_{\odot}$ DD-formed pulsar processes.

\begin{tabular}{lc}
\hline Process & $\begin{array}{c}\text { Timespan } \\
t_{\min }<t<t_{\max }\end{array}$ \\
\hline Bi-lobed to spheroidal CE & $\sim-2,000$ ? years \\
Chandrasekhar-mass WD formed & $\sim-2$ ? seconds \\
DD core-collapse to 2 ms pulsar & $\sim-0.5<t<0.5$ ? s \\
Gamma-ray burst ( $\ell$ GRB) & $\sim 0.5<t<\sim 100$ ? s \\
Pulsed GRB afterglow & $\sim 0.5 \mathrm{~s}<t<1$ day \\
Ejection spindown phase, $\theta_{V} \ll 1$ & $0<t<2$ years \\
Transition to $\theta_{V} \sim 1$ & $2<t<4$ years \\
GR-dominated spindown & $3<t<100$ ? years \\
Broadly beamed pulsations & $4<t<10$ years \\
Plasma depleted & $10<t<\infty$ years \\
Cas A-type thermal X-ray source & $10<t<400$ years \\
Magnetic dipole-dominated spindown & $100<t<\infty$ years \\
\hline
\end{tabular}

angle, which may have modified the resulting jet into the approaching and retreating conical features now easily visible in the HST ACS images [7]. The high-velocity cloud, HVC A0 [51], may be an example of a pulsar-driven jet long separated from its progenitor.

Eventually, as the plasma continued to thin with time, its maximum density just outside of $R_{\mathrm{LC}}$, occurred between here and $2 R_{\mathrm{LC}}$, resulting, because of (1), in pulsations driven close to the pulsar's rotational equator, and according to our self-consistent solution, in the line of sight to the Earth. Such a beam cannot possibly produce the observable excess luminosity that may have been seen by 1991 [52-54], as the amount of ${ }^{57} \mathrm{Co}$ required to otherwise account for the excess $\left(m_{V} \sim 17.4 \sim 275 \mathrm{~L}_{\odot}\right)$ was only barely consistent with hard X-ray and infrared spectral data $[55,56]$. If we were in the cusp of the pulsar within SN 1987A during this epoch, because of the distance ${ }^{-1}$ law associated with this direction, the implied luminosity would have dropped from $10 \mathrm{~L}_{\odot}$ (roughly magnitude 21 ), by half the distance modulus to the LMC, some 9.4 magnitudes, or a factor of $\sim 5,000$ to $\sim 0.002 \mathrm{~L}_{\odot}$. Moving from $10 \mathrm{pc}$ to $\sim 12 \mathrm{lt}-\mathrm{d}$ $(0.01 \mathrm{pc})$, the likely distance to the equatorial band (velocity $\sim 2,500 \mathrm{~km} \mathrm{~s}^{-1}$ ), the apparent luminosity falls by another factor of 1,000 , far short of the required excess, and all possible other beams cannot possibly make any difference. Because our geometrical solution puts the pulsar behind the equatorial torus visible in ACS images taken years later, the excess luminosity may have been due to an accretionpowered X-ray flux, reprocessed within ejecta thinning with time.

A few years earlier, it was unlikely that the $2.14 \mathrm{~ms}$ signal would have been detectable in the broadband, as limits established in early 1988 [31] are comparable to levels of the $2.14 \mathrm{~ms}$ signal observed in the I band between 1992, Feb. and 1993, Feb. The 2.14 ms pulsar candidate was last detected in 1996, Feb. [24], and by 2002 there was no evidence of any such source in ACS images, which only really means that any pulsar within SN 1987A had entered the "Cas A" phase, ${ }^{8}$ having exhausted its surrounding excess plasma and perhaps also because the Earth was no longer in the "cusp" of its beam(s). Still, the central source should turn on when the pulsar encounters matter from time to time. Thus, continued observations are warranted.

\section{The SN 1987A Link to GRBs}

Without (or perhaps even with) the $\mathrm{H}$ and $\mathrm{He}$ in the envelope of the progenitor of $1987 \mathrm{~A} \mathrm{Sk}-69^{\circ} 202$, the collision of the jet with the 1987A polar ejecta (which produced the early light curve and Mystery Spot) might be indistinguishable from a full $\ell$ GRB [6]; otherwise, it would just beg the question of what distant, on-axis such objects would look like. It is also the case that few or no $\ell$ GRBs have been found in elliptical galaxies, and that the DD process must dominate (as always, through binary-binary collisions), by a large factor, the neutron star-neutron star mergers in these populations, even when requiring enough white-dwarf-white dwarf-merged mass to produce core collapse. All of these facts/realizations lead to the alternate conclusion that the DD process may produce sGRBs in the absence of common envelope and polar ejecta, the means by which they would otherwise become $\ell$ GRBs. If the sGRBs in ellipticals are due to mergers of white dwarfs, we can conclude that (1) the pre common envelope/polar ejecta impact photon spectrum of $\ell$ GRBs may be well characterized as those of sGRBs, (2) sGRBs are offset from the centers of their elliptical hosts possibly because they are white dwarf-white dwarf mergers to core-collapse in their hosts' globular clusters (to produce most of their ms pulsars [57], and (3) neutron star-neutron star mergers may not make GRBs as we know them and/or be as common as previously thought. Thus, sGRBs, which last a few seconds [58], may not flag neutron star-neutron star mergers, which may last only a few $\mathrm{ms}$, the same timescale as the $30-\mathrm{Jy}, \mathrm{DM}=375$ radio burst [59].

Supernova 1987A, with its beam and jet producing its early light curve and Mystery Spot, is therefore potentially the Rosetta Stone for three of the four types of GRBs: $\ell$, i, and s GRBs $[60,61],{ }^{9}$ with both polar ejecta and common envelope, red supergiant common envelope and no polar ejecta, and neither polar ejecta nor common envelope, respectively [62].

In addition to axially driven pulsations, the SLIP model makes the very remarkable prediction that the component of pulsar intensity which obeys (1) diminishes only as distance $^{-1}$ (see appendix), and verified experimentally [63, 64], ${ }^{10}$ and has been dramatically confirmed [23], and noted by others [65], for pulsars in the Parkes Multibeam Survey (e.g., [66]) with single narrow pulses (see Figure 10 and Section 6.1). ${ }^{11}$ There is also evidence that GRBs and their afterglows share this characteristic $[67,68]$, which supports the SLIP prediction of axially driven pulsations when plasma is available at many $R_{\mathrm{LC}}$. The SLIP prediction also explains how GRBs and their afterglows can be visible across the Universe. As a consequence of this prediction, we initiated a campaign of high speed monitoring of GRB afterglows in 2008 and 2009 at the Lick Observatory Crossley 36 inch telescope (without success- "live" bright afterglows occur 


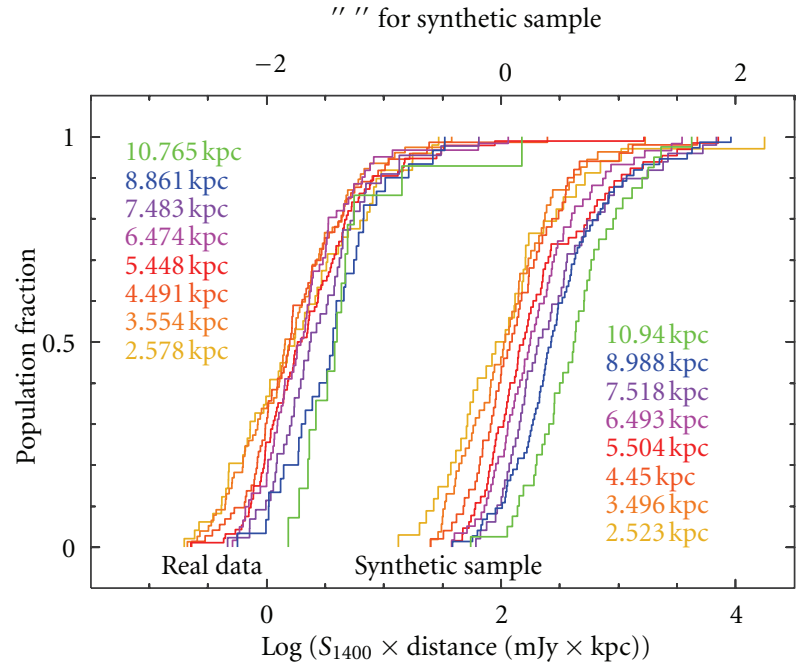

FIGURE 10: Left, lower horizontal axis: the population of 497 pulsars from the Parkes Multibeam Survey which fall into binned distances, with centers between $\sim 2.5$ and $\sim 10.75 \mathrm{kpc}$, plotted against the log of the product of their $1400 \mathrm{MHz}$ fluxes and their NE2001 distances from $[69,70]$. Right, upper horizontal axis: the same for a synthetic distribution of pulsars which obey the inverse square law. The two families of curves are both in order of increasing distance from left to right at a population fraction of 0.2 .

about one night in 60) and will reinitiate it as soon as the opportunity presents itself.

If, as for SN 1987A, the vast majority of $\mathrm{SNe}$ are DD initiated, then, by measuring the pulse period, $P$, of the optical/near infrared pulsations from an afterglow and assuming the pulsars resulting from DD are all produced at a standard spin period, $P_{0}$, first measured from SN 1987A near $2.14 \mathrm{~ms}$, but corrected to near $2.00 \mathrm{~ms}$ because of material ejected, the redshift is given by

$$
z=\frac{P}{P_{0}}-1
$$

and even a moderately precise $P$ (by standards) may yield a very precise redshift.

SLIP predicts that the peak of the emission for slowest pulsars occurs in the gamma-ray band [71-73], and this is supported by recent gamma-ray detections of slow $(\sim 1 \mathrm{~Hz})$ pulsars in supernova remnants by FERMI (e.g., [74]). Without this feature, one would naively question whether SLIP could produce pulsed GRB afterglows when the predicted post-core-collapse strength of the magnetic field at $100 \mathrm{~s}$ of $R_{\mathrm{LC}}$, whether from a several teragauss $80 \mathrm{~Hz}$ pulsar or a few gigagauss $500 \mathrm{~Hz}$ pulsar, would be well below the few megagauss necessary to drive cyclotron radiation in the optical and near-infrared bands. SLIP is more complicated; the centripetal acceleration of the polarization currents leads to contributions for a wide range of high frequencies. The essential necessary ingredient is plasma (see Section 6.2), and in this situation, there's plenty.

There is no requirement in the SLIP model on the rotator being a neutron star-a white dwarf will do as long as it

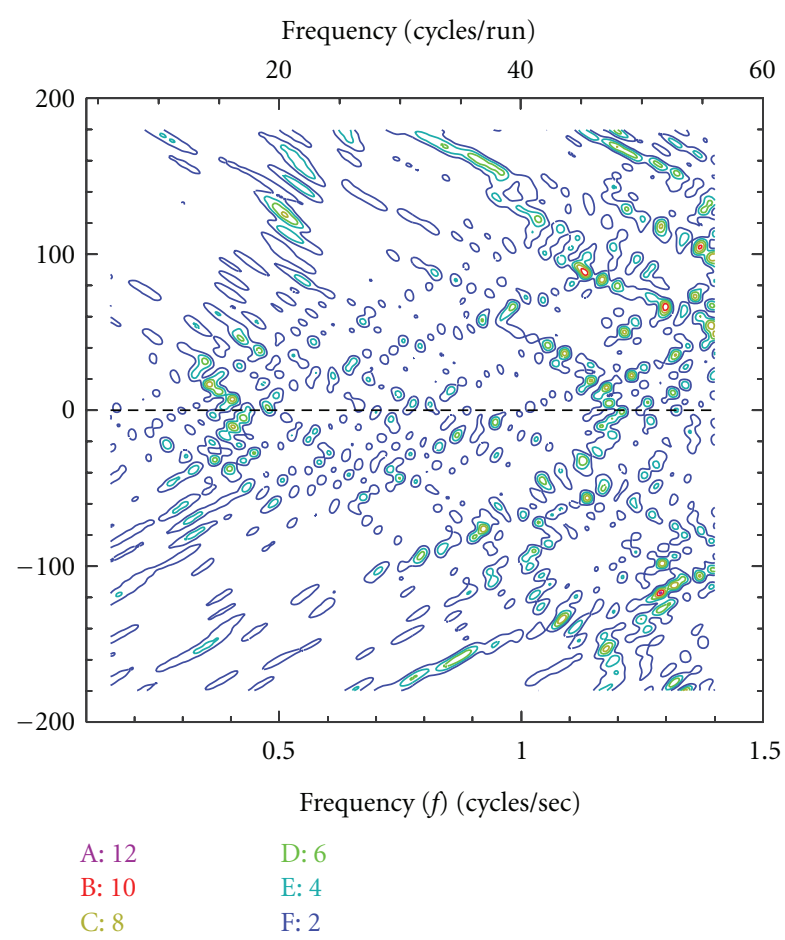

FIGURE 11: Low frequency Fourier power from GRB 960216 (with $\sim 18,000$ events in $40 \mathrm{~s}$ ) is contoured on the frequency-frequency derivative plane.

has a magnetic field and there is plasma outside of its light cylinder, whether this comes from pulsar-generated plasma sheets or gravitationally attracted ISM or SNR plasma. If this is the case, strongly magnetized pulsars may not make GRBs, and it might even be possible for a precore collapse, $\sim 1.4 \mathrm{M}_{\odot}$ white dwarf, spinning at its minimum period of $1.98 \mathrm{~s}$, to emit the prompt part of a GRB, and, as with the afterglow, the distance ${ }^{-1}$ law would likewise ameliorate the energy requirement, even with the large expected spinup. This also raises the intriguing possibility that a GRB could be produced without core collapse, and a large spin down may be present.

We tested for spinup/down in the GRB with the highest fluence in the BATSE catalog, 960216 [75], by Fourier, transforming the first $40 \mathrm{~s}$ of events and contouring power on the frequency- $(\partial f / \partial t)$ plane. Power appears in Figure 11, though not significant without further confirmation, at a mean frequency of $0.50 \mathrm{~Hz}$ and derivative of $+0.08 \mathrm{~Hz} \mathrm{~s}^{-1}$ and also for spinup/down about an order of magnitude smaller in the 0.35 to $0.42 \mathrm{~Hz}$ region (the results for higher frequencies, assuming $0 \dot{f}$, are also negative). Bursts with even better statistics (perhaps from FERMI) may be necessary to further test this hypothesis.

It is difficult for a GRB produced by pulsations during spinup to produce an inverted distance law because of the changing $v$. Alternately, GRBs may be produced when gamma-ray photons from the initial collimated SLIP photon flux scatters off of circumstellar material such as polar ejecta [76]. For material such as polar ejecta, in a plane 
perpendicular to a line of sight from the SN proper, the pattern of illuminated, scattering, concentric rings expands supraluminally, and thus, this emission also exhibits a distance $^{-1}$ law for certain directions. However, the initial gamma rays from the compact remnant actually penetrate the polar ejecta considerably, and thus, the scattering produces a volume source. What will be observed as a GRB across the Universe will be whatever produces a supraluminal source with an inverted distance law.

The geometric model with small-angle scattering of gamma rays in distant polar ejecta can explain other details of $\ell$ GRBs such as their $\sim 100 \mathrm{~s} \mathrm{~T}_{90}{ }^{\prime} \mathrm{s},{ }^{12}$ the negligible spectral lag for late $(\sim 10-100 \mathrm{~s})$ emission from "spikelike" bursts [77], and why "precursor" and "delayed" contributions have similar temporal structure $[62,78]$.

Moving a $10^{40} \mathrm{erg} \mathrm{s}^{-1}$ source from $25 \mathrm{lt}$-d to 8 billion light-years $(z \sim 1)$, assuming a distance ${ }^{-1}$ law, would only reduce the apparent magnitude to -15 . Optical flashes of visual magnitude 2, which reddened as they faded, were observed on two occasions by Howard Bond (private communication, 2008). No known triggers occurred at the noted times.

X-ray afterglows of GRBs are characterized by a power law index, $\alpha$, for the decline of intensity with time and another, $\beta$, to characterize the power law decline of the $\mathrm{X}$-ray spectrum. The temporal index is discontinuous at sudden changes in slope called "jet breaks". The two indices can be related through the "closure relations" for various circumstances affecting the jet, though with limited success for Swift GRBs [79]. In the SLIP model, breaks in the temporal slope of the optical afterglows, if they existed, would be caused by sudden changes in plasma density outside of the neutron star through (1). Because the steep decline of the Xray flux $(\alpha \sim 3)$ matches that of the GRB, which is unlikely to be pulsed, we do not expect the X-ray afterglow to be pulsed either, and their jet breaks may be caused by changes in the scattering of high-energy photons due to structure in the polar ejecta [76]. However, in order to be observable at all, the X-ray afterglows must have an inverted distance law similar to GRBs resulting from a supraluminal process of one sort or another. Whether or not these are pulsed at all remains to be determined.

SLIP has little difficulty explaining the unusually long duration of GRB 101225A ( $\left.\mathrm{T}_{90}>2000 \mathrm{~s}\right)$ through a coalescence between a neutron star and another star [80, 81], that is, a "borrowed SN". Initially, the outer layers of the star will be ejected by SLIP, and this activity will become progressively more violent with the inspiral of the neutron star as plasma becomes available at increasing $R_{\mathrm{LC}}$, though the tilt of the rotation axis relative to the plane of the inspiral will matter. It is this progression that is unusual for the GRB and is responsible for the longevity through gamma rays scattering in previously ejected material. A magnetar or, again, the formation of a black hole is unnecessary to make the jets (and highly unlikely unless the star is extremely massive). This is yet another case where high time resolution observations are sorely needed.

\section{Double Degenerate in Type Ia/c SNe}

Since 2007, Feb., it appeared unavoidable that Type Ia SNe were also DD generated, one of the causes being the long list of reasons why Ia's cannot be due to thermonuclear disruption $[62,82-85]$. Now, with two out of two $\mathrm{SNe}$ Ia (SNR 0509-675 and SN 2011fe), leaving no symbiotic companion [86, 87], it is not clear if this ever happens in any progenitor, and empty $\mathrm{SN}$ remnants almost always contain a neutron star which is not visible, just as the one in Cas A is barely visible [88]. Further, this means that Ia's and Ic's (these latter have been regarded by many as DDinitiated neutron star producing since the invention of the classification) are both due to the DD process. Thus, these must form a continuous class: Ia's when viewed from the merger equator (with lines of $\mathrm{Fe}$ ); and Ic's when viewed from the merger poles, because this view reveals lines of the $r$ process elements characteristic of Ic's, ${ }^{13}$ part of the reason for the differing spectroscopic classification and the high approaching velocities (e.g., "hypernovae") ${ }^{14}$ due to the view looking down the the axis of the approaching bipolarity.

In the application of Type Ia SN luminosities for cosmological purposes, the increase in blue magnitude from the light curve maximum, $\Delta M_{B}$ (essentially an inverse measure of the width of the light curve in time), measured in the first few weeks of SN Ia proper time, is used to correct the Ia luminosity for the variable amount of ${ }^{56} \mathrm{Ni}$ produced [89]. However, the direct relation, between the $\Delta M_{B}$ of the widthluminosity relation and the fractional SiII polarization in Ia's, pointed out by [90], is more meaningfully interpreted as an inverse relation between the SiII polarization and luminosity (unlike the Fe lines, SiII lines must also exist in the axial features because they are also observed in Ic's, and their polarization in Ia's is a result of the more rapid axial extension when viewed close to the merger equator).

This inverse relation would be expected in Ia's if the luminosity of the (very extended and productive of polarization) axial features were fixed, while the luminosity of the (less extended and less productive of polarization) toroidal component is driven by the amount of encapsulated ${ }^{56} \mathrm{Ni}$ positron annihilation gamma rays, which can be very high. As with 1987A-like events, it would again beg the question of "What else they could possibly be?" and "delayed detonation" [91] or "gravitationally confined detonation" [92] and may not produce polarization which would be inversely proportional to luminosity. And unless the view is very near polar, this geometry can produce split emission line(s) on rare occasions, as was seen in SN 2003jd [93], and thus again, there is no need to invoke exotica or an entire population (III) to account for GRBs [94, 95].

Because there is a spectroscopic difference between Ia's and Ic's, the SLIP-driven polar jets are either deficient in ${ }^{56} \mathrm{Ni}$ or are too diffuse to encapsulate their gamma rays, or both. No observation of any recent SN other than SN 1986J, $2006 \mathrm{gy}$, and 2007bi, and including all ever made of Type Ia $\mathrm{SNe}$, is inconsistent with the bipolar geometry of 1987A.

All this raises serious concerns about the use of $\mathrm{SNe}$ Ia in cosmology, because many Ia/c's in actively star-forming galaxies belong to the continuous class, and some of these 
and most Ia's in ellipticals, may not encapsulate a sufficient fraction of their gamma rays to be bolometric, especially given the toroidal geometry, lying as much as two whole magnitudes (or more for nearly naked core collapse) below the width-luminosity relation (the faint SNe Ia of [96]). Thus the $\log$ of the systematics (2+ magnitudes) can be an order of a magnitude bigger than the $\log$ of 1 plus the small effect (0.25 magnitude) from which dark energy was inferred in the same sense, an effect which could easily swamp Malmquist bias, the largest systematic working in the opposite sense.

In the SLIP model, the pulsar eviscerates its stellar remnant as long as there is remnant remaining; the $\mathrm{SN}$ cannot "close in" to a spherical configuration until much later when it will essentially become a shell source. This enforces a toroidal geometry of ejecta near its rotation equator, which may not conform to the width-luminosity relation because of unencapsulated positron annihilation gamma rays from the toroid, an assertion supported by the early detection of hard X-rays and gamma rays [97100]. However, the local sample was selected on the basis of conforming to the width-luminosity (W-L) relation, which is by definition [101] the high end of the luminosity function. Thus, the unavoidable inclusion of faint Ia's in the distant sample leads to a bias which makes it appear to be anomalously dim, assuming a distance ${ }^{-2}$ law, which seems appropriate for these objects. Supernovae which do not conform to the $\mathrm{W}-\mathrm{L}$ relation have also been noted in 2005, by [102], for high redshift ESSENCE SNe.

\section{Sco X-1, LMXBs, SS 433, and Other Details}

6.1. DD and SN Luminosities. The double-degenerate mechanism ensures that many $\mathrm{SNe}$ are born from the core collapse of a postmerger white dwarf with a rotation period near $2 \mathrm{~s}$. In this circumstance, the amount of ${ }^{56} \mathrm{Ni}$ produced depends on how much and what type of material is left in the common envelope in excess of the $\sim 1.4 \mathrm{M}_{\odot}$ lost to the neutron star (and emitted neutrinos), which can exceed this. The paltry amounts of ${ }^{56} \mathrm{Ni}$ associated with Ib's and at least $90 \%$ of IIs are likely the result of dilution of their thermonuclear fuel with $\mathrm{He}$ and/or $\mathrm{H}$ due to the $\mathrm{DD}$ merger process. ${ }^{15}$ Thus, SNe 2006gy and 2007bi [103, 104] do not have to be pair-instability SNe [105-108] or the former of a collision of two massive stars [109]; only massive FeSNe of up to $75 \mathrm{M}_{\odot}$, which may actually have produced 3 and $6 \mathrm{M}_{\odot}$ of

${ }^{56} \mathrm{Ni}$, respectively, and a strongly magnetized neutron star remnant, a prediction which, in the case of SN 2006gy, can be tested soon. ${ }^{16}$ Alternately, if the progenitor of SN 2007bi was stripped of its $\mathrm{H}$ and $\mathrm{He}$ by some mechanism, it could have been a DD (Type Ia/c) SN with a high luminosity due to a high mass $(\mathrm{C}$ and $\mathrm{O}$ ) in its common envelope.

6.2. SLIP, Plasma, and Intermittent Pulsations. The presence of plasma makes a huge difference to rapidly rotating, weakly magnetized neutron stars. Strong transient pulsations have occurred during observations of SN remnants or X-ray binaries which have never been subsequently confirmed, and yet have no explanation other than as a real astrophysical signal (e.g., the Cygnus Loop, see [110]). Judging from the high fraction of empty SN remnants, the population of "quiet" neutron stars must exceed all other "loud" populations combined [111]. All Galactic pulsars have the interstellar medium from which to draw plasma with their intense gravitational field, but frequently this is not enough; then only when such a neutron star encounters a cloud of matter will it become sufficiently luminous to be detected. It is for this reason that observations of SN 1987A should continue, as has already been suggested in Section 3, in spite of conclusions drawn from recent "snapshot" null results $[34,112]$.

SLIP also makes no assumptions about the carrier wave - a physical process such as cyclotron radiation/strong plasma turbulence is necessary for the pulsar to radiate at shorter/longer wavelengths, but centripetal acceleration of the emitting region further modifies this spectrum by adding emission to shorter wavelengths. Thus, whether or not emission is observed in a band where SLIP predicts it should exist, as, for example, the few GeV cutoff of the Fermi pulsars [113], if real, would indicate something about the physical conditions of, and/or near, the pulsar.

In the context of the SLIP model, emission from rotation powered pulsars is not haphazard-radiation from the known pulsars may very well be detected from Earth because we are "in the cusp," that is, we are in the part of the pulsar's beam that decays inversely only as the first power of distance (1), which may be one of the reasons why repeated attempts to detect pulsations from extragalactic SNe have failed (e.g., [114]; another reason might be that all except SN 1987A were too distant).

6.3. Low-Mass X-Ray Binary (and Other) Rotation Rates. Although SLIP makes the detection of pulsations from the luminous LMXBs unlikely (see Section 1), there may still be a few ways to tell fast that their neutron star components are spinning. Because neutron stars are the accreting compact members of most, if not all LMXBs [115], thermonuclear (Type I) X-ray bursts are common, as are the oscillations which occur during them $[116,117]$. Some of the oscillation frequencies are quite high, with $1122 \mathrm{~Hz}$ for XTE J1739-285 topping the list [118]. Many of these frequencies are thought to reveal the actual rotation period of the neutron star. Many, including the first observed burst oscillations in the optical pulsation in 4U1254-69 [119], show oscillations with only modest frequencies (in this case, $36.4 \mathrm{~Hz}$ ), ${ }^{17}$ which also might be the neutron star rotation rates. These modest spin rates are consistent with the LMXB rotation rates settling to a rate where spindown from SLIP, which may increase more than linearly with mass transfer rate, can exceed by several orders of magnitude any spinup due to accretion, which is subject to the Eddington limit. The association with rotation periods might be made when these are observed to remain the same (or very close) in repeated bursts. Pulsations from LMXB rotation frequencies may be observed in those for which the restrictive beaming $\left(\theta_{V} \ll 1\right)$ occasionally relaxes, that is, for which plasma is restricted to fewer $R_{\mathrm{LC}}\left(\theta_{V} \cong 1\right.$, see Section 6.10). 
There is also a small group of about a dozen transient accreting millisecond X-ray pulsars, most of which also only show oscillations during Type I bursts (e.g., [120]). Many of these have accretion rates that are not persistently high and also have very high spin rates such as $400 \mathrm{~Hz}$ for SAX J1808.4-3658. In addition, of the three fastest pulsars, two, J1748-2446ad and J1959+2048, are currently evaporating their extremely low mass companions and one, B1937+21, already has. All this is consistent with an inverse relation, at some point between the rates of mass transfer and net rates of spinning up.

6.4. Sco X-1. The dual radio lobes of Sco X-1 [121], which expand at $\sim 0.3-0.57 \mathrm{c}$, and to which energy must be transported at speeds of $0.95 \mathrm{c}$ or greater, are a nearly exact match to the derived kinetics of the Mystery Spot of SN 1987A and might have actually been observed and detected from 1987A in the radio band, given a sufficiently large and sensitive radio array in the Southern Hemisphere, which did not exist at the time. Wright et al. [122] have noted that the $<19$-hour orbital period constrains the companion to a very faint $\mathrm{M}$ dwarf or a white dwarf stellar core. This would imply that the accretia would be rich in metals as is the case with SN 1987A, though the $0.95 c$ transport of energy in its jets also argues for some hydrogen, or at least spall protons (see Section 6.7), which are then accelerated by the usual SN "boost" mechanism of heavier elements hitting lighter ones. If so, then considering that the elements of the accretia may not be stratified, as they may have been at least partially so within the newly born remnant of SN 1987A, it is amazing that this mechanism works at all, and this, plus the relative dearth of $\mathrm{H}$, may explain the relative weakness of Sco X-1's very fast jet component.

6.5. Historic Remnants and Pulse Profiles. If we are not "in the cusp," for any annulus of plasma beyond the pulsar light cylinder, whose induced radiation is free to propagate to infinity, spinning neutron stars may only appear as thermal sources such as the one in Cas A [88]. A century older, as is the case with Tycho 1572, not even the thermal sources were detectable. However, the extinction of $A_{V}=$ $3.47,+0.13,-0.29$ mag. to the Kepler 1604 remnant [123], though a revision up from 2.2 [124], is still much less than the $6.2 \pm 0.6 \mathrm{mag}$. to Cas A [125], with the distances nearly equal at 3.2 and $3.4 \mathrm{kpc}$. Thus, assuming that extinction traces $\mathrm{H}$ column and all $\mathrm{SNe}$ produce compact remnants, the pulsar in this remnant should be visible as a thermal source, so perhaps the neutron star in Cas A is visible because of SLIP beaming. Evidence does linger, however, even in the outwardly very spherical remnant of SN 1006 as bipolar high-energy emission in XMM and VLA images [126], with the high-velocity chimneys seen in Cas A [127] being long gone. For many pulsars, such as the Crab, which were discovered because of an SN remnant association, we may not be in the "in the cusp," and thus, the isotropic optical luminosity of the pulsar may currently still be $\sim 3 \mathrm{~L}_{\odot}$. However, for those pulsars that are in the cusp, the SLIP model predicts that the pulse profile will consist of two very close peaks, ${ }^{18}$ which would seem to exclude the Crab (e.g., $[128,129])$ and ascribed the interpulse to some other mechanism [130] (see the appendix), but the solitary pulse of the $16.1 \mathrm{~ms}$ pulsar, J0537-6910, will split, if allowed by the fitting process (see immediately below).

6.6. The Pulsar, J0537-6910, and the SLIP Model. SLIP predicts that pulsed emission arises outside of the light cylinder, either from current sheets originating close to the pulsar or from ISM plasma which has been concentrated by gravity from the neutron star, or both. In either case, the plasma outside of the light cylinder is most dense just outside of the light cylinder. In the case of the $62 \mathrm{~Hz} \mathrm{X}$-ray pulsar in the Large Magellanic Cloud, J0537-6910, SLIP explains why it is not a strong optical pulsar [131]. Although J0537 only has an effective dipole field of $\leq 1 \mathrm{TG}$, since it is an oblique rotator [30], its actual surface field could be as strong as that of the Crab, B0540-693, 3-5 TG, or even stronger. In addition, because of its rapid $62-\mathrm{Hz}$ spin, the magnetic field just outside of its light cylinder, which is less than half as distant as that of the Crab pulsar, is an order of magnitude larger still. This means that the cyclotron frequencies just outside of the light cylinder of J0537 are an order of magnitude (or more) higher than those which contribute to the optical pulsations of the Crab, and thus are shifted out of the optical regime upward toward the X-ray regime. Since cyclotron frequencies do not have subharmonics, and the centripetal acceleration at the light cylinder, $\omega \mathrm{c}$, is also higher than those of the Crab or B0540, J0537 cannot and does not produce strong optical pulsations However, assuming that the frequency 2 nd derivative persists at $-0.95 \times 10^{-21} \mathrm{~Hz} \mathrm{~s}^{-2}$, J0537 will spin at $31 \mathrm{~Hz}$ in $\sim 3,800$ years and may be a strong optical pulsar by that time.

As mentioned above, the single narrow peak of the pulse profile of J0537 is likely an indication that we observe its Xray pulsations at least partially (the pulse width is $\sim 10 \%$ of the period) as a result of the distance ${ }^{-1}$ law, and its $2-10 \mathrm{keV}$ pulsed X-ray luminosity could be lower than $2 \times 10^{35} \mathrm{erg} \mathrm{s}^{-1}$ as estimated by [132].

6.7. The Positron Excess, WMAP "Haze", and Fermi "Bubbles". A beam of protons, with kinetic energies of up to $2.2 \mathrm{GeV}$ or greater, will eventually produce electrons with similar energies. Electrons/positrons with even higher energies may result from photon scattering because the cross section varies inversely as the square of the particle mass and/or the leptons may, in turn, be further accelerated by magnetic reconnection, in woundup magnetic fields near the Galactic center, or other mechanisms [133], possibly to $\mathrm{TeV}$ energies, to produce the WMAP "Haze"/FERMI "Bubbles" observed in that direction $[134,135]$. In addition, the SNR loss/relativistic injection of ${ }^{56} \mathrm{Ni}$ positrons into the pulsar jet, which occurs because of the bipolarity of $\mathrm{SNe}$ and which also makes them unfit for easy cosmological interpretation, may show up as an excess in cosmic ray data [136-138]. This may offer a satisfying resolution for the apparent anomalous dimming of distant SNe explained in terms of local cosmic-ray abundances, without invoking the creation 
of electron-positron pairs near pulsars and their acceleration along "open" field lines which do not exist if one believes in the validity of the Sommerfeld equations $[17,18]$. TeV fermions generated by $\mathrm{SNe}$ and/or pulsars would alleviate the short lifetime problem $\left(\sim 10^{6} \mathrm{yr}\right)$ associated with the Galactic center as a potential direct source. SLIP does not require pulsars to generate positrons in order for them to emit radiation, so the $10-100 \mathrm{GeV}$ positron flux may not be dominated by contributions from local pulsars but may be provided instead by the supernovae that gave birth to them recently. The main difficulties lie in accounting for how and why the excess rises at energies above $10 \mathrm{GeV}$ and below $100 \mathrm{GeV}$.

Protons at multiple $\mathrm{GeV}$ energies will destroy the (relatively stationary) heavy nuclei they collide with, resulting in the release of free neutrons which can drive the $r$-process in supernovae. A density of $10^{24} \mathrm{~cm}^{-3}$ in free neutrons has been invoked to drive this process, but SLIP can provide this near the center of the newly formed remnant. However, SLIP also clearly drives the $r$-process, whose element lines are visible in early Ic spectra, at the poles of $\mathrm{SNe} \mathrm{Ia/c}$, as described in Sections 1 and 5, where the influence of annuli well outside of the stellar core bypasses that same core to produce focused beams at the two poles, where $\mathrm{C}$ and $\mathrm{O}$ are both available. Detailed calculations are deferred for future efforts (see, e.g., [139]).

6.8. The SS 433 Jets. The extreme collimation of the initial beam and jet in SN 1987A and even the strong $\mathrm{H} \alpha$ seen in its spectrum near day 19.6 are suggestive of a similar situation in SS 433 (e.g., [140]), and a rapidly spinning pulsar has been proposed for the underlying central engine (e.g., [141]), although mass estimates for the compact object vary from 0.8 to $16 \mathrm{M}_{\odot}[142-145]$. The big problem with SS 433 is accounting for the mechanical power necessary to produce $10^{34} \mathrm{erg} \mathrm{s}^{-1}$ of $\mathrm{H} \alpha$ from recombination in two jets with velocities of $0.26 c$, which could amount to $10^{42} \mathrm{erg} \mathrm{s}^{-1}$ if each atom has only one recombination. Because of the few minute variability of the $\mathrm{H} \alpha[146,147]$ with no variability of its velocity $>1 \%$, the standard method of its solution is to invoke a clumpiness of $10^{5}$ in the jets titled "bullets" (e.g., $[148])$, so that each atom recombines many times $\left(\sim 10^{2.5}\right)$.

Although [149] constrained the parameter space for the bullets, assuming the $\mathrm{H} \alpha$ production mechanism was an interaction of the jets with a B-star wind, the possibility of many excitations per atom, due to beamed radiation, was an admitted exception to the requirement for bullets. Of course this is exactly what SLIP does in the process, easily producing the kinematic and collimation properties of the SS 433 jets from an ms pulsar buried in hydrogen-dominated accretia, where $\mathrm{L} \infty$ at the source is redshifted to $\mathrm{L} \alpha$ in the mature jets (this is exact for $\beta=0.28$, but the actual $\beta$ is lower [0.26], consistent with the finite bandwidth necessary for the jet acceleration mechanism to work), the usual SN boost mechanism being unavailable because of the lack of high $Z$ accretia.

If SLIP turns out to be a necessary mechanism to produce the jets and their $\mathrm{H} \alpha$ flux, then the rarity of SS 433 may be less, due to the wind from a Population I B star, than its association with a weakly magnetized ms pulsar, which clearly did not originate from a massive solitary star similar to what we think describes the primary. The best chance to detect a pulsar in SS 433 may be during a low state, should that ever happen, there having been no sign of any pulsar (e.g., [150]) in the high state it has occupied since the discovery of its bizarre behavior in 1978. A 2 ms pulsar could power the jets for $10^{4}$ years if each atom produces $\sim 20 \mathrm{H} \alpha$ photons, not even accounting for any spinup due to accretion. It is more likely, however, that the spin period of SS 433 is larger because a $2 \mathrm{~ms}$ period is associated with the evisceration of SN progenitors by SLIP, and, in the presence of plasma at many $R_{\mathrm{LC}}$ as happens in both SS 433 and $\mathrm{SNe}$, will have a very large spindown, whereas the actual spin of SS 433 will settle to a rate where spinup from accretion is balanced by spindown due to SLIP. This rate will likely be a bit higher than what is typical for the luminous LMXBs, perhaps closer to a period of $5 \mathrm{~ms}$, because of the lack of heavy elements loading SLIP (see Section 6.10).

6.9. Star Formation Avalanche in the Early Universe. Computer simulations of clustering in the Early Universe fail without the addition of some extra agent to increase the rate at which clusters form (see, e.g., [151]). The problem is that the agents which can be easily inserted into the codes, such as dark matter or dark energy, almost always involve controversial physics which may not be valid. Aside from such agents, the only known mechanism which is frequent and violent enough to possibly play the same role are the pulsar-driven jets resulting from the supernovae of the first stars.

Although high velocity material will suppress star formation locally (M.-M. Mac Low, 2011, private communication), even the early Universe $(z \sim 7)$ is vast, and this same material will initiate star formation [152-155] at some distance from the pulsar in the two initially polar jets. Without spreading, the Mystery Spot column, $\sigma_{\mathrm{MS}}$, of $0.5 \mathrm{gm} \mathrm{cm}^{-2}$ will penetrate even the early Universe at $z=7$, with $4 \%$ of the present day critical density in baryonic mass. Assuming, for the sake of simplicity, a linear (conical) spreading scale of $\ell_{0}=3 \times$ $10^{16} \mathrm{~cm}$ in an interstellar medium of density of $\rho_{\mathrm{B} 7}=1.9 \times$ $10^{-28} \mathrm{~g} \mathrm{~cm}^{-3}$ (4\% of critical density at $z \sim 500^{1 / 3}-1 \sim 7$ ), and solving for the pathlength, $\ell$, whereby a column of mass equal to the spread-diluted Mystery Spot column has been swept up in its last $10 \%$, we get

$$
\ell \sim 200 \mathrm{pc}\left(\frac{\ell_{0}}{3 \times 10^{16}}\right)^{2 / 3}\left(\frac{\sigma_{\mathrm{MS}}}{0.5}\right)^{1 / 3}\left(\frac{\rho_{\mathrm{B}}}{1.9 \times 10^{-28}}\right)^{-1 / 3} .
$$

The collimation of the Mystery Spot is not exactly known, but it cannot be as extreme as the $10^{4 \rightarrow 5}$ of the initial beam/jet. However, it could easily amount to a few square light-days at a distance of 10 light-days. In that case, the plume would be about a few light years across at $100 \mathrm{pc}$. Along the way, shearing vortices may initiate star formation, with higher velocities at the larger distances from the endpoint.

The actual situation may be much more complicated, as the decline of the jet collimation with time will impact 
the previously formed vortices. The stars formed by this process may also initiate another round of star formation as they move through the primordial ISM and so on, with each cluster retaining a tiny, though important, fraction of the initial jet velocity. Infant clusters in close proximity with nearly matched velocities may merge due to their sustained mutual gravitational attraction. In this way, the SLIP jets may initiate a star formation avalanche, possibly leading to minicluster and/or stream formation in a few million years, with no requirement for dark matter. The recent discovery by GALEX $[156,157]$ of velocities between close pairs of distant galaxies, which do not reflect their mutual gravitational attraction, may be the result of the separation velocities of pulsar-driven jets in their formation of star clusters. Again, detailed calculations are deferred for future efforts.

6.10. Pulsar "Recycling". Pulsars with rapid spins and weak magnetic fields are commonly referred to as having been "recycled," wherein these attributes are a result of a (usually) long dead pulsar undergoing an extensive interval of accretion from a binary companion which it acquired or already had, that as a result has increased its spin rate from the few-second period typical of a very old strongly magnetized pulsar to the ms period range, and as a side effect, either of the accretion or slow decay of the effective magnetic dipole by some other mechanism because of advanced age, or both, reduced its original several teragauss magnetic field by 2 to 4 orders of magnitude.

Here, however, we have seen evidence that the spindown due to SLIP can greatly exceed any spinup due to accretion. Thus, the only way to tell if a pulsar has been recycled is by a mass substantially in excess of $1.3 \mathrm{M}_{\odot}$ (as is the case for J1903+0327), ${ }^{19}$ but even then, there's no guarantee that the pulsar has suffered any net spinup, the result depending on accretion rate and history, spin rate, and magnetic field strength and configuration.

To gain further understanding of this process, 1723 pulsars are located on the log period-period derivative plane in Figure 12. On this plot, the connection between the "ordinary" pulsars in the upper right to the "recycled" pulsars in the lower left is a two-lane one-way highway. The top lane (higher-period derivative) contains all six of the globular cluster pulsars and nine Population I pulsars for the period range from 10 to $100 \mathrm{~ms}$, while the bottom lane contains just Population I pulsars for that range. Metalicity and gravitational contamination are unlikely contributors to this pattern. The lanes have numerical slopes of about 1.5, meaning that if pulsars migrate to the right and slightly up in the lanes, the period derivatives gain 3 parts for every 2parts-gain in period. By contrast, the same ratio is 3 to 1 for the young (4,000-year old) $16.1 \mathrm{~ms}$ PSR J0537-6910 (see Section 6.6), twice the slope of the two lanes (its effective magnetic dipole increases by $0.02 \%$ per year), so perhaps the pulsars in these lanes actually do migrate right and up along their respective sloped paths.

Unfortunately, none of the six globular cluster pulsars in the upper lane within that period range have measured second frequency derivatives (which should be negative

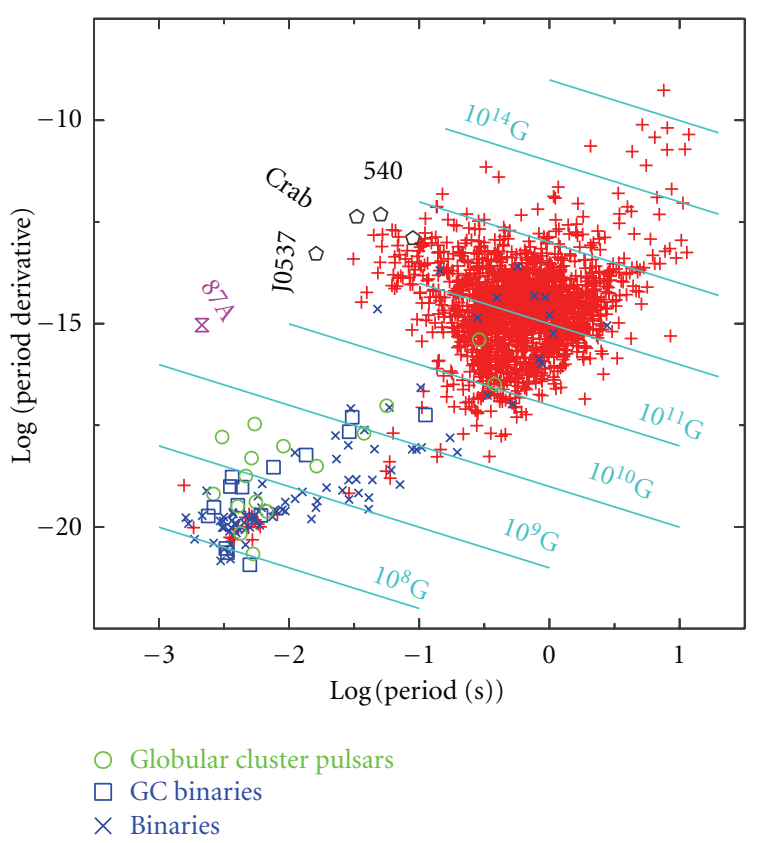

Figure 12: A plot of 1723 pulsars from the ATNF pulsar database in the log period-period derivative plane. Nonbinary, nonglobular cluster pulsars are marked by "+" signs, nonglobular binary pulsars by $\times$ 's, nonbinary globular cluster pulsars by circles, globular binary pulsars by squares, four strongly magnetized young pulsars by pentagons, and the $2.14 \mathrm{~ms}$ signal from SN 1987A by an hourglass.

for this migration but which will also suffer extremely from gravitational contamination), so we cannot test the hypothesis that these pulsars are migrating on the slightly upward path. Of the 32 nonglobular pulsars in the lanes within this period range, 2 have negative, and 2 have positive, frequency second derivatives, so half of these four pulsars are not migrating within the lanes. With a period derivative of $1.88 \mathrm{e}-20, \mathrm{~J} 1903+0327$ lies on the low boundary of the short-period end of the upper lane. The offset between the two lanes corresponds to nearly two orders of magnitude in period derivative, but only one order of magnitude in field strength.

Still, this offset between the two lanes is likely to be due to different accretion histories, provided, accretion does in fact reduce pulsar effective dipoles as postulated by $[158,159]$. The simplest interpretation is that pulsars in the upper lane, containing about ten of eleven globular cluster pulsars with periods $>8 \mathrm{~ms}$, have never suffered accretion and were born fast and weakly magnetized.

It is even difficult to argue in favor of a strongly magnetized origin for those pulsars in the lower lane, as it would seem strange that this population starts only from those "ordinary" pulsars with the smallest period derivatives, and neither from the much larger population of "dead" pulsars with even lower period derivatives. ${ }^{20}$ It may be more likely that this (lower) lane is from faster pulsars which accreted from binary companions to become LMXBs that have ceased to accrete, and are left there. Similarly, the upper lane may populate the main group just above its lowest point. 
So on average these pulsars slowed during their phase of high accretion, which is in line with the modest QPO frequencies seen in many LMXBs, ${ }^{21}$ and the high frequencies seen from most of the accreting millisecond X-ray pulsars, which have very low accretion rates.

As the accretion rate diminishes when the binary companion begins to withdraw from its Roche limit, for whatever reason as long as this is a gradual process, the pulsar will have an era of spinup, possibly resulting in the pileup at 35 ms periods. Here, however, pulsars are found with period derivatives still lower than those of the lower path, thus requiring a population of neutron stars which were born only weakly magnetized and likely spinning in the millisecond range.

Thus, these two discrete lanes connecting the "normal" pulsar group in the upper right to the ms pulsars in the lower left require a source of pulsars whose spin rates are faster than $200 \mathrm{~Hz}$. Again, the most likely source to populate these paths is the source of fast-born $2 \mathrm{~ms}$ pulsars from the DD process. Since there are orders of magnitude more neutron stars generated by the DD mechanism than by Fe catastrophe, there should be a much larger reservoir of "dead" weakly magnetized pulsars than their "dead" strongly magnetized cousins. A study of the high-space velocities of solitary neutron stars as compared to the masses and low eccentricities of double neutron star binaries has also reached the conclusion of the necessity of two mechanisms to produce neutron stars [160], the DD process being the second (though overwhelmingly predominant) mechanism.

\section{Conclusion}

We have derived a self-consistent solution for the onset $(\sim 11 \mathrm{lt}-\mathrm{d})$, and depth $(\sim 13 \mathrm{lt}-\mathrm{d})$, of the polar ejecta of the progenitor of SN 1987A, the energetics of its beam/enhanced UV flash $\left(>10^{40} \mathrm{erg} \mathrm{s}^{-1}\right)$, the kinetics of its jet $(\beta \sim 0.95$ for $\left.\sim 10^{-6} \mathrm{M}_{\odot}\right)$, and the angle from the line of sight to the Earth $\left(\sim 75^{\circ}\right)$. There is plenty of evidence for the absence of any strongly magnetized pulsar within SN 1987A, and such a pulsar may not have the rotational energy to account for the kinetics derived anyway. Thus, we have argued, through the paradigm of a model of pulsar emission (SLIP) from polarization currents updated supraluminally beyond the pulsar light cylinder [20], that SN 1987A, its beam/jet, "Mystery Spot," and possible $2.14 \mathrm{~ms}$ pulsar remnant, are intimately related to SS 433 , Sco X-1, and as many as $99 \%$ of LMXBs, GRBs, ms pulsars, and other $\mathrm{SNe}$, including all Type Ia SNe. The SLIP model explains, in a natural way (1), the changes over time observed in the collimation of the $\mathrm{SN}$ 1987A beam and jet.

The time lags, energetics, and collimation of $\ell$ GRBs are consistent with those of 1987A's initial beam, jet, and the inferred polar ejecta. When the bipolarity of SN 1987A is interpreted through this model, its pulsar clearly had ablated part of the $\sim 10 \mathrm{M}_{\odot}$ of ejecta, eviscerating the remnant by blowing matter out of its poles at speeds up to $0.95 c$ or greater and enforcing a toroidal geometry on the remaining equatorial ejecta. Since there is no reason to suggest that this is not universally applicable to all $\mathrm{SNe}$, this geometry has grave implications for the use of Type Ia SNe as standard candles in cosmology (see, e.g., [161] and Section 5). Lines observed from Type Ic SNe provide proof that SLIP beams drive the r-process at the rotational poles, in or near the progenitor photosphere (Sections 1, 5, and 6.7). No other model can explain why such lines are only observed in $\mathrm{SNe}$ Ic (which are actually SNe Ia when observed away from the poles, and also when the $r$-process lines can not be observed) the reason being that $\mathrm{C}$ and $\mathrm{O}$ at the stellar surface are only available in $\mathrm{SNe} \mathrm{Ia/c.}$

Because even weakly magnetized neutron stars buried within plasma can spin down at a rate which exceeds their ability to spin up from accretion by orders of magnitude, pulsars interacting with the rest of the remnant progenitor (if this rest exists) clearly cannot be ignored-they are the driving force behind the disruption of their progenitor stars in supernova explosions, in spite of the difficulty of detecting them within the remnant, now explained by the associated beaming. This has observable consequences; the start of the associated UV flash will show up within minutes, or even seconds, of the core collapse and neutrino flux, and the timetable of many SN 1987A events needs to be revised accordingly. In addition, the existence of a robust mechanism for disrupting progenitor stars means that the stellar mass at which core collapse continues on to create a black hole is much larger than previously thought-perhaps closer to $75 \mathrm{M}_{\odot}$ or even higher.

There appears to be no need to invent exotica to explain GRBs- the SLIP model provides the young pulsar (or even a near-Chandrasekhar-mass white dwarf) with an initially tightly collimated beam as the central engine and makes the very specific and testable prediction that GRB afterglows are, in fact, pulsars usually spinning at $500 \mathrm{~Hz}$ in the proper frame.

Beaming has always been a wild card for pulsars, and for the SLIP model, the beaming is effectively infinitepart of the radiation decays only as the first power of the distance, and the two cones of this beam (which subtend a solid angle of measure 0) will change in polar angle as plasma conditions change outside of the pulsar light cylinder. Beams and jets which are less than maximally collimated (those which originated from annuli of less than maximal radii) may not have enough concentrated energy to break through extensive surrounding material and rather help to lift it away from the neutron star. Rotating objects for which pulsations are not detected may simply be beamed in other directions. Thus, the disappearance, or persistent absence, of pulsations from rotating compact objects such as those which might lie within the Cygnus Loop (Section 6.2), SN 1987A (Section 2), SS 433 (Section 6.8), and Sco X-1 (Section 6.4) and other LMXBs (Section 6.3) does not automatically imply that there never was a pulsar and/or that the compact object is a black hole the energy requirement for a pulsar being negligible because of the distance ${ }^{-1}$ law. Finally, it may be that all transient events observed in the very distant Universe, including the outbursts of QSOs, are seen because they have a similar inverted distance law, which is a result of a supraluminal excitation of one sort or another. 
Such restrictive beaming $\left(\theta_{V} \ll 1\right.$ and plasma at many $R_{\mathrm{LC}}$ ) explains, in a natural way, the nondetections of pulse frequencies from the luminous LMXBs, and less restrictive beaming $\left(\theta_{V} \cong 1\right.$ and plasma at few $\left.R_{\mathrm{LC}}\right)$ the intermittent detections of oscillations from the less luminous LMXBs - the accreting millisecond X-ray pulsars. The neutron star components of the LMXBs will settle to a spin rate where spinup from accretion, which has an absolute limit, balances spindown from SLIP losses, which increases with mass transfer rate almost without limit. Thus, the neutron star spin rate in LMXBs will vary inversely with luminosity (see Sections 6.3 and 6.10).

As is the case for many other things, the devil in this Universe may lie in the details. Thus, while the most extensive simulations of the early Universe still require dark matter to form galaxies (see, e.g., $[162,163]$ ), the jets driven by the pulsars resulting from the $\mathrm{SNe}$ of the first stars may provide the perturbations necessary to initiate the next generation of star formation in such a way as to include much of this generation in rapidly growing star clusters and/or streams (see Section 6.9). Thus, although it may take longer to form the first stars without dark matter, the formation of clusters via pulsar-driven jets may make up this time. The peculiar motions observed from distant galaxy pairs $[156,157]$, which would otherwise be expected to show motions reflecting their mutual gravitational attraction, may be due to the pulsar jetstar-formation process.

In addition, because of the large systematic effects involved in measuring Type Ia (and all other) SN luminosities due to the unsuitability of their geometries (see Section $5,[96,102])$, we simply cannot tell if they suffer any anomalous dimming with cosmological distances without further understanding of the $\mathrm{SN}$ process and the ability to apply this understanding to carefully select the distant sample. This was the only really direct evidence that the expansion of the Universe is accelerating, and now the scientific community must again question the existence of dark energy. Without it, in addition to the possible lack of the need for dark matter in the formation of star clusters, there may be no longer any numerical coincidence to support the role of dark matter in concordance cosmology. Recent observations of solitary ring galaxies have also cast significant doubt on the existence of dark matter $[164,165]$.

Thus, aside from bizarre entities such as quantum entanglement currents, there is not much evidence for any dark "stuff" present in the recent Universe $(z \leq 1)$ having any observable effect, and this has been due in significant part to a new understanding of the role of pulsar-driven jets. Still, the evidence is strong that $\Omega_{\text {total }}=1$ at the era of recombination $(z \sim 1000$, e.g., [166]). However, in addition to kinematic redshift, gravitational redshift becomes important when looking back that far, and our knowledge of gravity is incomplete at best. Perhaps the best assessment of this apparent factor of $\sim 20-25$ difference in $\Omega_{\text {total }}$ between the era of recombination and the more recent Universe is that it remains a deeper mystery.

The path to the intimate and productive study of the $\mathrm{SN}$ and GRB processes is now clear; with high-time-resolution observations of GRB afterglows, through the selection effect of the inverted distance law operating across billions of light years, we let the Universe reveal the behavior of the responsible pulsars. It might appear that a Universe without the need for dark matter or energy, collapsars, hypernovae, pair instability $\mathrm{SNe}$, super-Chandrasekhar-mass white dwarfs, frequent collisions of massive stars, and neutron star-neutron star mergers which make sGRBs is much less "exotic" than previously thought. However, pulsars, that is, clocks and minutes-old neutron stars to boot, which can be seen across almost all of this same Universe (which is never in equilibrium), including even core-collapse remnants of the first stars, and which may yield practically instant redshifts just from their pulse frequencies alone, may well suffice in explaining all of the issues which gave rise to the previously mentioned entities, are more in line with Occam's Razor and are also, of themselves, extremely worthy of study.

\section{Appendix}

\section{A Short SLIP Primer}

Models of pulsar emission usually involve the motion of charged particles between the neutron star and the light cylinder. The outer gap model [167] produces the emission by particle acceleration in the gap just outside of the last closed magnetic field line within the light cylinder and the null surface $(\boldsymbol{\Omega} \cdot \mathbf{B}=0)$. The polar cap models involve pair production, electron acceleration, and production of secondaries close to the magnetic poles of the neutron star [168]. Other models produce emission via inverse Compton upscattering of lower energy photons (see, e.g., [169] and references therein). In the SLIP model, radiation from polarization currents induced beyond the light cylinder initially focuses on the light cylinder and drives a particle wind along with a beam. SLIP is indifferent to material within the light cylinder and for isolated pulsars relies on material from the ISM concentrated by the gravitational field of the neutron star (see, e.g., [73]).

Electromagnetic radiation can be generated by a timedependent polarization, $\mathbf{P}(t)$, just as it can from currents of free charges, $\mathbf{j}$, displacement, $\mathbf{D}$, and electric field, $\mathbf{E}[170]$ as follows:

$$
\nabla \times \mathbf{H}=\frac{4 \pi}{c} \mathbf{j}+\frac{1}{c} \frac{\partial \mathbf{D}}{\partial t}=\frac{4 \pi}{c}\left(\mathbf{j}+\frac{\partial \mathbf{P}}{\partial t}\right)+\frac{1}{c} \frac{\partial \mathbf{E}}{\partial t},
$$

where $\mathbf{H}$ is the magnetic field and $c$ is the speed of light in vacuo. Although the motion of the charged particles in the polarization currents is, in general, slow, there is no limit to the speed at which they can be modified, similar to a wave produced by fans in a stadium at a sports event. In particular, the action of a rotating dipole, such as a neutron star with a nonaligned magnetic field, can change polarization currents much faster than the speed of light at many light cylinder radii, $R_{\mathrm{LC}}$.

As the (mostly dipole) magnetic field of the neutron star rotates, much of the polarization current field beyond $R_{\mathrm{LC}}$ will be instantaneously parallel because the magnetic field in opposite directions with respect to the axis of rotation is also opposite. Thus, the polarization currents can be thought of 


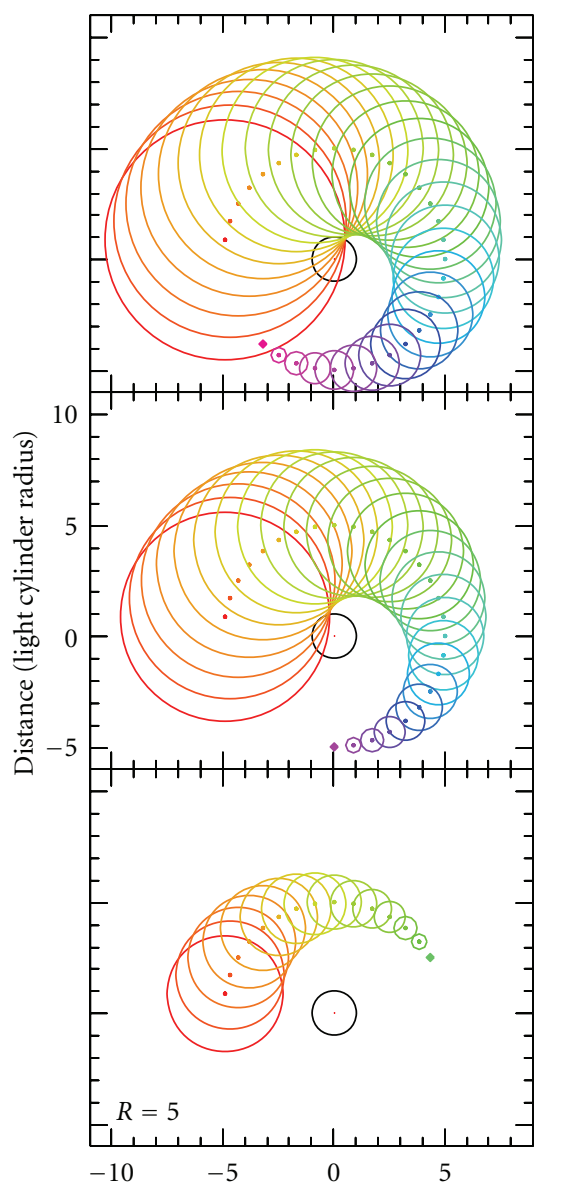

Figure 13: Bottom to top: a progression of Huygens' wavelets for a supraluminal source moving at 5 times the speed of light, with 36 wavelets in a full circle. The light cylinder footprint is marked by the small black circle.

as a single vector function of radius, which is rotating rigidly with the pulsar rotation frequency, $\omega$.

The influence of currents from an annulus of a given radius, on a point on the light cylinder and in the same plane perpendicular to the axis of rotation, will then consist of the magnetic field produced by such currents and the influence of the cyclotron radiation and strong plasma turbulence induced by the currents. However, the influence of the currents is affected, in a nontrivial way, by their centripetal acceleration in the supraluminally rotating frame of reference (see, e.g., [71]), a complication which we will ignore in this discussion. The net influence on the point can at least be understood by the influence of a progression of a series of Huygens' wavelets, as shown in Figure 13 which shows many wavelets intersecting a point tangent to the light cylinder. Ultimately, the wavelets from nearly a third of a whole revolution will intersect at such a point still further clockwise around the light cylinder. More than this would be unproductive, both because directions of further contributions to $\mathbf{H}$ at the point would be more than $60^{\circ}$

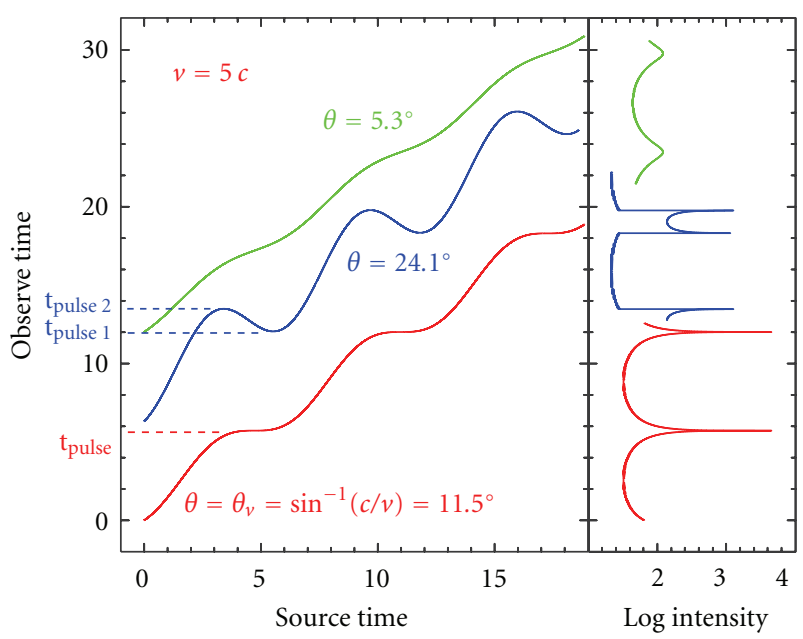

FIGURE 14: Left: observer time as a function of source time for a supraluminally induced circular excitation at 5 times light speed with a period of $2 \pi$ for three polar directions. Delays in the observer time of order millions have been suppressed, and the three curves have been arbitrarily offset by a few units for clarity. Right: the integral, into 512 discrete bins per cycle of observer time phase, of source time dwell versus observer time for the three curves (i.e., pulse profiles).

away from the mean phase, and, of course, the induced timevarying cyclotron radiation or strong plasma turbulence would also suffer out-of-phase contributions.

As time progresses further, this focus point, or "cusp", will split as it moves out of the plane, spiralling out on two cones of polar half angle given by (1) (see, e.g., $\theta_{P}$ of equation (13) of [22]). The dependence of observer time on source time for three different $\theta_{P}$ and the three different resulting pulse profiles are shown in Figure 14. The singly peaked pulse profile (repeated once) for the direction corresponding to the "cusp" for this $v=5 \mathrm{c}$ case (polar angle of $\sim 11.5^{\circ}$ ) is shown in the lower part of the right frame. Almost exactly half of the $\sim 1,000$ pulsars in the Parkes Multibeam Survey have a singlepulse peak with a full width at half maximum less than 3\% of the pulse period (see, e.g., [49] and Section 4), and SLIP predicts that all of these will be very closely spaced doubles (see Section 6.5).

For larger polar angles, the pulse profiles are obviously double and less sharp (right frame, middle); for smaller polar angles (right frame, top), the pulse profiles are again single, but still smaller, and eventually not sharp at all. The advantage in the intensity of one pulse profile over another will depend on how many points are used in plotting the curves - the more points, the greater the advantage, until the limiting case of the distance ${ }^{-1}$ law is reached. The point of inflection in the lowest curve of Figure 14 is the circular analog of a shockwave, and it is this that results in the distance ${ }^{-1}$ law.

If the rotation-powered pulsars which radiate from just beyond the light cylinder (see Section 6.6) produce the characteristic singly peaked pulse profile and a beam which, according to (1), is very nearly equatorial, how is it that 
pulsars with doubly peaked profiles find the phase space in greater polar angle to generate those profiles?

One possible production mechanism for complex pulse profiles is a multipole component of the neutron star magnetic field. Barring that, the Crab pulsar may offer a clue to this conundrum. Its interpulse, which alone is similar to the small polar angle top pulse profile of Figure 14, shows the emission bands in the $2-10 \mathrm{GHz}$ range predicted by SLIP while its main pulse does not [130]. The possibility that the main pulse is a result of a less homogeneous environment and the bands are smeared out to uniformity seems to be excluded by these observations. Thus, the Crab's main pulse may arise from a different location, that is, a different radius, which would also imply emission from a radius greater than just outside the light cylinder, which in turn could mean plasma sheets generated closer to the pulsar travelling outward through $R_{\mathrm{LC}}$. Still, the equal $180^{\circ}$ swing in polarization across both the main and interpulse supports a SLIP process for both.

The movie of the Crab [26] in Chandra X-rays appears to show at least one nearly equatorial fan of pulsations and $0.5 \mathrm{c}$ wind, as predicted by SLIP through (1) for material just outside the light cylinder, with our line of sight between the lower rotation axis and the lower near-equatorial wind component. Weisskopf et al. [171] interpret this as a single circular feature projected onto the plane of the sky and offset from the plane of the sky containing the pulsar as a fan beam of some 4.5 degrees off the equatorial plane of the pulsar, which would indicate that the location of the SLIP emission occurs at 1.003 times the light cylinder radius. It is not clear if there is another fan, as predicted by SLIP, which does not appear because its aspect produces less back scattering. The width of the bottom lip of the "bell" supports a two-fan interpretation of some sort and may imply that plasma around the Crab pulsar does not extend a large fraction beyond $R_{\mathrm{LC}}(\sim 1,600 \mathrm{~km})$, although it has to exist more densely at great distance to reveal the very distant dominant ring.

Because our line of sight to the pulsar is much closer to the pole than the fan in the opposite hemisphere from that which produces the circular arc, the Crab's interpulse may be a small polar angle feature similar to the pulse profile in the upper right of Figure 14. There is the possibility that plasma is trapped at a few $R_{\mathrm{LC}}$ and leads to an emission fan that is more nearly polar, but just not easily visible in the movie, and could be associated with a second arc visible to the right (west) of the innermost arc. The fan in the opposite hemisphere to the one that produces this second arc may actually lie at, or closer to, the pole than our line of sight, and this would ease the angular requirement for producing sharp and double pulses and may, in some way, reveal the production mechanism of the Crab's main pulse.

The movie also shows that the Crab has polar jets, and there are two possible sources of supraluminal excitations of the same concentric feature which reveals the fan of wind and pulsations. The first supraluminal excitation may be due to the discrete waves of particles, made famous by the movie, impacting the concentric features. Although the timing of the particle wave arrivals at all azimuths will not be exact, it may be close enough to drive substantial polar jets. The second supraluminal excitation is due to the actual $\sim 30 \mathrm{~Hz}$ pulsations themselves and for these, the timing will be exact because there will frequently be enough material in the features to trace the path of each individual pulse over a wide range of azimuth, in spite of the fact that they are of order $100 \mathrm{~s}$ of millions of light-pulse periods away ( $\mathrm{a}$ few light-months).

If pulsars on the "cusp" have to be very close to satisfying (1), it may not take a lot of range in polar angle closer to $90^{\circ}$ to populate the doubly or multiply peaked sample. Those pulsars with smaller polar angle may be relatively suppressed due to the poor detectability of their bland singly peaked pulse profiles (the top right curve in Figure 14). Further studies of artificial pulsar populations may yield a definitive answer to this question about the distribution of types of pulse profile.

\section{Acknowledgments}

The author would like to thank Dr. John Singleton for supporting this work through the Los Alamos National Laboratory (LDRD) Grant no. 20080085DR, "Construction and Use of Superluminal Emission Technology Demonstrators with Applications in Radar, Astrophysics, and Secure Communications," and Grant no. 201100320ER, "Novel Broadband TeraHertz Sources for Remote Sensing, Security, and Spectroscopic Applications" as well as Drs. Joe Fasel, Todd Graves, Bill Junor, and Andrea Schmidt. The author is also grateful to Larry Earley of Los Alamos, Jim Johnson and Carl Pennypacker of Lawrence Berkeley Laboratory, John Saarloos for loans of equipment for use at Lick Observatory as well as Keith Baker, Kostas Chloros, Elinor Gates, Bryant Grigsby, Rem Stone and the rest of the entire staff of of Lick Observatory for their assistance, and M. T. "Brook" Sanford of Los Alamos for programming assistance. I also wish to thank Joel Roop and Andy Toth of Keithley Instruments for the loan of an engineering unit of the KUSB 3116 for testing at home while far away from Lick Observatory. This work was performed under the auspices of the Department of Energy.

\section{Endnotes}

1. Because not all pulsars show the characteristically sharply peaked pulse profiles which indicate that they are observable because they obey the distance ${ }^{-1}$ law, some pulsars are indeed observed from an angle, $\theta \neq \theta_{V}$.

2. No observation of any GRB afterglow has yet been made in high time resolution, but they are expected to be highly pulsed because of the associated inverted distance law (see Section 4). However, the afterglows of GRBs 990510,990712 , and 020405 were measured to be $1.7 \%$, $2.9 \%-1.2 \%$, and $10 \%$ linearly polarized [172-175], which could be a signature of pulsed emission. The prompt emission of GRB 041219 was also found to be highly and variably linearly polarized by INTEGRAL 
[176], though we do not expect such emission to be pulsed.

3. The CTIO V band center occurs at 5,500 $\AA$, as opposed to $5,100 \AA$ for the FES, and in consequence, the FES magnitudes have been diminished by 0.075 in Figure 3 to account for the resulting luminosity offset. In addition, comparison of the CTIO data [8] with the FES data at the minimum near day 7 and the decrement near day 20 reveals that the published CTIO times may be too early by 1 day and have been adjusted in this work. All other significant disagreements between the fluxes can be explained by temporary spectral features appearing in one band and not in the other.

4. This $\mathrm{SN}$, in the edge-on spiral galaxy, NGC 0891, exceeds the luminosity of the Crab nebula at $15 \mathrm{GHz}$ by a factor of 200 [177], and thus is thought to have occurred because of a core collapse due to iron photodissociation catastrophe $(\mathrm{FeSN})$, producing a strongly magnetized neutron star $\left(\sim 10^{12} \mathrm{G}\right.$ - the origin of magnetic fields in neutron stars is still poorly understood, though it is believed that thermonuclear combustion in a massive progenitor to an Fe core is related).

5. Attempts to get around the discrepancy between the cluster ms pulsar population and their very much-less numerous X-ray binary-supposed progenitors, invoke a short time interval with a very high spinup rate, ostensibly produced by a very high accretion rate in the $\mathrm{X}$ ray binaries, to generate their ms pulsars. There are two problems with this hypothesis. The first is that relatively slowly rotating recycled pulsars weighing $1.7 \mathrm{M}_{\odot}$, in the core-collapsed globular cluster, Ter 5 [178], have removed high accretion rate from contention as an alternative mechanism to produce the ms pulsars in the non-core-collapsed globular clusters without a longduration (and more obvious) X-ray source phase (see below regarding J1903+0327). The second problem is that we will see in Section 6.3 that because of SLIP, the relation between accretion rate and spinup rate is often inverse. The three ms pulsars in Ter 5, O, P, and ad [179], and five other non-GC pulsars with periods $<2 \mathrm{~ms}$, may have been recycled starting with periods near $2 \mathrm{~ms}$.

6. The $2.15 \mathrm{~ms}$ pulsar, J1903+0327 [180], has a main sequence companion in a highly eccentric $(\epsilon=0.44)$, 95-day, >105- $\ell$ t-s orbit, from which it could never have accreted mass, but its high mass of $1.67 \mathrm{M}_{\odot}$ [181] indicates an extended era of accretion, ostensibly from a closer companion which has since been evaporated by the pulsar. Whether this accretion converted a strongly magnetized only moderately rapidly spinning neutron star into J1903, at the same time drastically attenuating its magnetic field, rather than just converting a light J1903 into a heavier one, is still an unresolved question, given the effectiveness of weakly magnetized, rapidly spinning pulsars in driving jets, where jet-driven spindown might easily dominate accretion-driven spinup. Thus, J1903 might have been recycled from its lighter self to its heavier self with little net change in spin rate (see Section 6.10).

7. Because there is initially always too much angular momentum, this rate is set by the branching of the Maclaurin and Jacoby solutions, leaving a nearChandrasekhar-mass white dwarf spinning with a period of $1.98 \mathrm{~s}$.

8. Calculations with the SLIP model involve a variation of Kepler's equation, which relates the eccentric anomaly, $E$, to the mean anomaly, $M$, using the eccentricity, $\epsilon$, $E-\epsilon \sin E=M$, but in this case $\epsilon>1$. Such calculations are notoriously difficult, even for a compact star not surrounded by remnant plasma. Needless to say, no such calculations have been done to date, and thus, no calculation through the core-collapse process, done so far, possibly including those of collapsars, can possibly be valid. One side effect not properly accounting for the pulsar, and the large amount of ${ }^{56} \mathrm{Ni}$, when strongly magnetized pulsars are produced, is a very low estimate for the mass, $\sim 25 \mathrm{M}_{\odot}$, above which the core collapse continues on to a black hole. SNe 2006gy and 2007bi, each with several $\mathrm{M}_{\odot}$ of ${ }^{56} \mathrm{Ni}$, argue for a much higher transition mass.

9. All except soft gamma repeator (SGR) GRBs, which are estimated to amount to less than $5 \%$ of sGRBs and $1.5 \%$ of the total [182].

10. Anyone who has noticed the wildly varying acoustic signature from helicopters has experienced this directly $[183,184]$ and similar effects in other geometries are also well known in acoustics $[185,186]$.

11. The pulsars at high flux in Figure 10 neatly overlap when multiplied by their distances, confirming the distance ${ }^{-1}$ law, while those with low fluxes are detector limited and thus are ordered according to their distance. The synthetic sample was generated with a distance ${ }^{-2}$ law and are by contrast spread out according to their distance even for the parts with the highest fluxes/population fractions, well in excess of their detector-limited parts near the bottom at low fluxes.

The subbeams which make up the cusp radiation have a constant height parallel to the rotation axis $\left(\delta\left(\theta_{V}\right) \times\right.$ distance $=$ constant $)$, and thus, to conserve energy their flux drops only as distance ${ }^{-1}$. However, because they do have a constant height (of order of several $R>R_{\mathrm{LC}}$ ), the solid angle they subtend also decreases as distance ${ }^{-1}$, and thus energy is again conserved [22]. However, as long as pulsar survey sensitivities keep up with the distance $^{-1}$ law and their survey beams are always filled with productive target, such as a galaxy, they will always detect more new pulsars with distance.

12. An offset of $0.5^{\circ}$ at $10 \mathrm{\ell t}$-d corresponds to a $33 \mathrm{~s}$ delay.

13. If sufficient matter exists, in excess of that lost to core collapse, to screen the Ia thermonuclear products-a rare circumstance in elliptical galaxies the reason why Ic's are absent from these. 
14. These velocities, near $0.4 \mathrm{c}$, match those of the Mystery Spot.

15. Helium has been found where it was not expected in almost all well-studied Type II SNe.

16. Such amounts of ${ }^{56} \mathrm{Ni}$ can lift a lot of stellar material, thus, $40 \mathrm{M}_{\odot}$ in an $\mathrm{SN}$ remnant is no longer a reason to invoke "millisecond magnetars" as the dispersal mechanism $[187,188]$.

17. This may have happened with 4U1728-24, which showed strong $104 \mathrm{~Hz}$ optical pulsations for a short interval (B. A. Peterson and J. E. Nelson, private communication, 1977; if this is the rotation rate, then the $\sim 111$ s periodicity is due to some other mechanism, see, e.g., [189]). This result was never published because of its high implied energetics, and it was never reproduced. However, there is no other good explanation, given the single-photon-counting detector used, and SLIP can explain the energetics (an image of the source at the same time should also have confirmed this), and further development of survey telescopes could also detect such events.

18. These are so close that the singly peaked profiles of the vast bulk of radio pulsars could easily be such doubles without our knowing it (see appendix).

19. Given that the best estimate for the mass of Her X1 is $1.3 \mathrm{M}_{\odot}[190,191]$ and the likelihood that it has been accreting $10^{-9} \mathrm{M}_{\odot} \mathrm{yr}^{-1}$ from HZ Her for several hundred million years (with gaps [192]), the best estimate for the standard initial strongly magnetized neutron star mass is likely closer to $1.25 \mathrm{M}_{\odot}$, which is supported by the masses of the strongly magnetized pulsars, J0737-3039B at 1.2489(7) [193] and J1906 + 0746 at $1.248(18) \mathrm{M}_{\odot}[194,195]$, respectively (see also [196] and references therein). This could mean that the standard Chandrasekhar mass is closer to $1.35 \mathrm{M}_{\odot}$, unless it is actually higher for weakly magnetized progenitors, or that $0.05 \mathrm{M}_{\odot}$ is typically excluded from the core collapse for strongly magnetized neutron stars.

20. This assumes that the accretion cycle from these to the lower left is always hidden, that is, the accretion is never interrupted until the pulsars arrive near the pileup near $5 \mathrm{~ms}$ nor the "normal" population with higher period derivatives. We reject the hypothesis that this does happen during the accreting phase but a large fraction somehow fall, neatly into this well-focused lane once their accretion ceases.

21. As well as the $104 \mathrm{~Hz}$ optical signal observed from the LMXB 4U1728-24.

\section{References}

[1] W. P. S. Meikle, S. J. Matcher, and B. L. Morgan, "Speckle interferometric observations of supernova 1987A and of a bright associated source," Nature, vol. 329, no. 6140, pp. 608611, 1987.

[2] P. Nisenson, C. Papaliolios, M. Karovska, and R. Noyes, "Detection of a very bright source close to the LMC supernova SN 1987A," The Astrophysical Journal Letters, vol. 320, pp. L15-L18, 1987.

[3] P. Nisenson and C. Papaliolios, "A second bright source detected near SN 1987A," Astrophysical Journal Letters, vol. 518, no. 1, pp. L29-L32, 1999.

[4] M. J. Rees, "A relativistic jet from SN1987A?" Nature, vol. 328, no. 6127, p. 207, 1987.

[5] T. Piran and T. Nakamura, "Jets in supernova 1987A?" Nature, vol. 330, no. 6143, p. 28, 1987.

[6] R. Cen, "A possible lateral gamma-ray burst jet from SN 1987A," Astrophysical Journal Letters, vol. 524, no. 1, pp. L51L54, 1999.

[7] L. Wang, J. C. Wheeler, P. Höflich et al., "The axisymmetric ejecta of supernova 1987A," Astrophysical Journal Letters, vol. 579, no. 2, pp. 671-677, 2002.

[8] M. Hamuy and N. B. Suntzeff, "SN 1987A in the LMC. III. UBVRI photometry at Cerro Tololo," Astronomical Journal, vol. 99, no. 4, pp. 1146-1158, 1990.

[9] W. Wamsteker, N. Panagia, M. Barylak et al., "Early observations of supernova 1987 A with the International Ultraviolet Explorer (IUE)," Astronomy \& Astrophysics, vol. 177, pp. L21L24, 1987.

[10] J. W. Menzies, R. M. Catchpole, K. van Vuuren et al., "Spectroscopic and photometric observations of SN 1987athe first 50 days," Monthly Notices of the Royal Astronomical Society, vol. 227, pp. 39P-49P, 1987.

[11] I. J. Danziger, R. A. E. Fosbury, D. Alloin et al., "Optical spectroscopy of SN 1987A," stronomy \& Astrophysics, vol. 177, pp. L13-L16, 1987.

[12] P. Mészáros, "Gamma-ray bursts," Reports on Progress in Physics, vol. 69, no. 8, pp. 2259-2321, 2006.

[13] S. E. Woosley and J. S. Bloom, "The supernova-gammaray burst connection," Annual Review of Astronomy and Astrophysics, vol. 44, pp. 507-556, 2006.

[14] N. Mirabal, J. P. Halpern, D. An, J. R. Thorstensen, and D. M. Terndrup, "GRB 060218/SN 2006aj: a gamma-ray burst and prompt supernova at $\mathrm{z}=0.0335$," Astrophysical Journal, vol. 643, no. 2, pp. L99-L102, 2006.

[15] F. Bufano, E. Pian, J. Sollerman et al., "The highly energetic expansion of SN 2010bh associated with GRB 100316D," The Astrophysical Journal, vol. 753, pp. 67-79, 2012.

[16] A. I. MacFadyen and S. E. Woosley, "Collapsars: gamma-ray bursts and explosions in "failed supernovae"," Astrophysical Journal Letters, vol. 524, no. 1, pp. 262-289, 1999.

[17] L. G. Kuo-Petravic, M. Petravic, and K. V. Roberts, "Selfconsistent solution for an axisymmetric pulsar model," Physical Review Letters, vol. 32, no. 18, pp. 1019-1022, 1974.

[18] T. M. Braje and R. W. Romani, "Magnetospheric scattering and emission in millisecond pulsars," Astrophysical Journal Letters, vol. 550, no. 1, pp. 392-396, 2001.

[19] H. Ardavan, "The mechanism of radiation in pulsars," Monthly Notices of the Royal Astronomical Society, vol. 268, pp. 361-392, 1994.

[20] H. Ardavan, "Generation of focused, nonspherically decaying pulses of electromagnetic radiation," Physical Review E, vol. 58, no. 5, pp. 6659-6684, 1998.

[21] B. M. Bolotovskii and V. L. Ginzburg, "Reviews of topical problems: the vavilov-cerenkov effect and the doppler effect in the motion of sources with superluminal velocity in vacuum," Soviet Physics Uspekhi, vol. 15, pp. 184-192, 1972.

[22] H. Ardavan, A. Ardavan, J. Singleton, J. Fasel, and A. Schmidt, "Morphology of the nonspherically decaying radiation beam generated by a rotating superluminal source," 
Journal of the Optical Society of America A, vol. 24, no. 8, pp. 2443-2456, 2007.

[23] J. Singleton, P. Sengupta, J. Middleditch et al., "A maximumlikelihood analysis of observational data on fluxes and distances of radio pulsars: evidence for violation of the inverse-square law," Physical Review Letters. In press, http://arxiv.org/abs/0912.0350.

[24] J. Middleditch, J. A. Kristian, W. E. Kunkel et al., "A 2.14 ms candidate optical pulsar in SN1987A," http://arxiv.org/abs/ astro-ph/0010044.

[25] R. W. Hanuschik and J. Dachs, "The $\mathrm{H}-\alpha$ velocity structure during the first month of SN 1987a in the LMC," Astronomy \& Astrophysics, vol. 182, pp. L29-L30, 1987.

[26] J. J. Hester, K. Mori, D. Burrows et al., "Hubble Space Telescope and Chandra monitoring of the Crab synchrotron nebula," Astrophysical Journal Letters, vol. 577, no. 1, pp. L49L52, 2002.

[27] B. E. K. Sugerman, A. P. S. Crotts, W. E. Kunkel, S. R. Heathcote, and S. S. Lawrence, "A new view of the circumstellar environment of SN 1987A," Astrophysical Journal, vol. 627, no. 2, pp. 888-903, 2005.

[28] A. Patruno, J. M. Hartman, R. Wijnands, D. Chakrabarty, and M. Van Der Klis, "Accretion torques and motion of the hot spot on the accreting millisecond pulsar XTE J1807-294," Astrophysical Journal Letters, vol. 717, no. 2, pp. 1253-1261, 2010.

[29] L. Lindblom, B. J. Owen, and S. M. Morsink, "Gravitational radiation instability in hot young neutron stars," Physical Review Letters, vol. 80, no. 22, pp. 4843-4846, 1998.

[30] J. Middleditch, F. E. Marshall, Q. D. Wang, E. V. Gotthelf, and W. Zhang, "Predicting the starquakes in PSR J0537-6910," Astrophysical Journal, vol. 652, no. 2, pp. 1531-1546, 2006.

[31] C. R. Pennypacker, J. A. Kristian, J. Middleditch et al., "Limits on an optical pulsar in supernova 1987A," The Astrophysical Journal, vol. 340, pp. L61-L64, 1989.

[32] H. Ogelman, C. Gouiffes, T. Augusteijn et al., "Search for the optical signatures of a pulsar in SN 1987 A," Astronomy \& Astrophysics, vol. 237, pp. L9-L12, 1990.

[33] J. Kristian, "No pulsar in SN1987A," Nature, vol. 349, no. 6312, p. 747, 1991.

[34] G. J. M. Graves, P. M. Challis, R. A. Chevalier et al., "Limits from the Hubble space telescope on a point source in SN 1987A," Astrophysical Journal, vol. 629, no. 2, pp. 944-959, 2005.

[35] J. Middleditch, J. A. Kristian, W. E. Kunkel et al., "Rapid photometry of supernova 1987A: a 2.14 ms pulsar?" New Astronomy, vol. 5, no. 5, pp. 243-283, 2000.

[36] R. M. Bionta, G. Blewitt, C. B. Bratton et al., "Observation of a neutrino burst in coincidence with supernova 1987A in the large magellanic cloud," Physical Review Letters, vol. 58, no. 14, pp. 1494-1496, 1987.

[37] K. Hirata, T. Kajita, M. Koshiba et al., "Observation of a neutrino burst from the supernova SN1987A," Physical Review Letters, vol. 58, no. 14, pp. 1490-1493, 1987.

[38] P. Podsiadlowski and P. C. Joss, "An alternative binary model for SN1987A,” Nature, vol. 338, no. 6214, pp. 401-403, 1989.

[39] T. Morris and P. Podsiadlowski, "The triple-ring nebula around SN 1987A: fingerprint of a binary merger," Science, vol. 315, no. 5815, pp. 1103-1106, 2007.

[40] C. Fransson, A. Cassatella, R. Gilmozzi et al., "Narrow ultraviolet emission lines from SN 1987A-evidence for CNO processing in the progenitor," The Astrophysical Journal, vol. 336, pp. 429-441, 1989.
[41] N. Sanduleak, Contr. CTIO, 1969.

[42] H. E. Schwarz, "Polarimetry of SN 1987A," Astronomy \& Astrophysics, vol. 177, p. L4, 1987.

[43] M. Cropper, J. Bailey, J. McCowage, R. D. Cannon, and W. J. Couch, "Spectropolarimetry of SN 1987A-observations up to 1987 July 8," Monthly Notices of the Royal Astronomical Society, vol. 231, pp. 695-722, 1988.

[44] P. Barrett, "Multicolour Stokes' polarimetry of SN 1987A in the LMC," Monthly Notices of the Royal Astronomical Society, vol. 234, pp. 937-956, 1988.

[45] A. G. Lyne, A. Brinklow, J. Middleditch, S. R. Kulkarni, D. C. Backer, and T. R. Clifton, "The discovery of a millisecond pulsar in the globular cluster M28," Nature, vol. 328, no. 6129, pp. 399-401, 1988.

[46] F. Camilo, D. R. Lorimer, P. Freire, A. G. Lyne, and R. N. Manchester, "Observations of 20 millisecond pulsars in 47 Tucanae at 20 centimeters," Astrophysical Journal, vol. 535, no. 2, pp. 975-990, 2000.

[47] K. Chen, J. Middleditch, and M. Ruderman, "Low-mass Xray binaries and millisecond pulsars in globular clusters," Astrophysical Journal Letters, vol. 408, no. 1, pp. L17-L20, 1993.

[48] M. Gilfanov and Á. Bogdán, "An upper limit on the contribution of accreting white dwarfs to the type Ia supernova rate," Nature, vol. 463, no. 7283, pp. 924-925, 2010.

[49] R. N. Manchester, G. B. Hobbs, A. Teoh, and M. Hobbs, "The Australia telescope national facility pulsar catalogue," Astronomical Journal, vol. 129, no. 4, pp. 1993-2006, 2005.

[50] P. Ghosh and F. K. Lamb, "Disk accretion by magnetic neutron stars," The Astrophysical Journal, vol. 223, pp. L83L87, 1978.

[51] G. L. Verschuur and D. L. Nidever, "The distance and stability of the HI filament that comprises HVC A0," Bulletin of the American Astronomical Society, vol. 43, 2011.

[52] R. Cowan, Supernova's Light Curve Baffles Scientists, Science News, 1991.

[53] N. B. Suntzeff, M. M. Phillips, D. L. Depoy, J. H. Elias, and A. R. Walker, "The late-time bolometric luminosity of SN 1987A," Astronomical Journal, vol. 102, no. 3, pp. 1118-1134, 1991.

[54] N. B. Suntzeff, M. M. Phillips, J. H. Elias, D. L. Depoy, and A. R. Walker, "The energy sources powering the late-time bolometric evolution of SN 1987A," Astrophysical Journal Letters, vol. 384, no. 2, pp. L33-L36, 1992.

[55] D. M. Rank, P. A. Pinto, S. E. Woosley et al., "Nickel, argon and cobalt in the infrared spectrum of SN1987A: the core becomes visible," Nature, vol. 331, no. 6156, pp. 505-506, 1988.

[56] S. Kumagai, T. Shigeyama, K. Nomoto et al., "Gamma rays, Xrays, and optical light from the cobalt and the neutron star in SN 1987A," The Astrophysical Journal, vol. 345, pp. 412423, 1989.

[57] N. Gehrels, C. L. Sarazin, P. T. O’Brien et al., "A short $\gamma$ ray burst apparently associated with an elliptical galaxy at redshift z $=0.225$," Nature, vol. 437, no. 7060, pp. 851-854, 2005.

[58] B. M. S. Hansen and M. Lyutikov, "Radio and X-ray signatures of merging neutron stars," Monthly Notices of the Royal Astronomical Society, vol. 322, no. 4, pp. 695-701, 2001.

[59] D. R. Lorimer, M. Bailes, M. A. McLaughlin, D. J. Narkevic, and F. Crawford, "A bright millisecond radio burst of 
extragalactic origin," Science, vol. 318, no. 5851, pp. 777-780, 2007.

[60] I. Horváth, L. G. Balázs, Z. Bagoly, F. Ryde, and A. Mészáros, "A new definition of the intermediate group of gamma-ray bursts," Astronomy and Astrophysics, vol. 447, no. 1, pp. 2330, 2006

[61] I. Horvath, Z. Bagoly, L. G. Balazs, G. Tusnady, and P. Veres, "SWIFT and BATSE bursts' classification," http://arxiv .org/abs/0912.3724.

[62] J. Middleditch, "The SN, 1987A link to others and gammaray bursts," http://arxiv.org/abs/0708.2263.

[63] A. Ardavan, W. Hayes, J. Singleton, H. Ardavan, J. Fopma, and D. Halliday, "Experimental observation of nonspherlcally-decaying radiation from a rotating superluminal source," Journal of Applied Physics, vol. 96, no. 8, pp. 4614-4631, 2004.

[64] A. Ardavan, W. Hayes, J. Singleton, H. Ardavan, J. Fopma, and D. Halliday, "Corrected Article: Experimental observation of nonspherically-decaying radiation from a rotating superluminal source," Journal of Applied Physics, vol. 96, no. 12, pp. 7760-7777, 2004.

[65] D. R. Lorimer, "Radio pulsar populations," in High-Energy Emission From Pulsars and Their Systems: Proceedings of the First Session of the Sant Cugat Forum on Astrophysics, Astrophysics and Space Science Proceedings, pp. 21-36, Springer, Berlin, Germany, 2011.

[66] D. R. Lorimer, A. J. Faulkner, A. G. Lyne et al., “The parkes multibeam pulsar survey-VI. Discovery and timing of 142 pulsars and a galactic population analysis," Monthly Notices of the Royal Astronomical Society, vol. 372, no. 2, pp. 777-800, 2006.

[67] D. A. Kann, S. Klose, B. Zhang et al., "The afterglows of Swiftera gamma-ray bursts. I. Comparing pre-Swift and Swiftera long/soft (type II) GRB optical afterglows," Astrophysical Journal Letters, vol. 720, no. 2, pp. 1513-1558, 2010.

[68] D. A. Kann, S. Klose, B. Zhang et al., "The afterglows of swiftera gamma-ray bursts. II. Type I GRB versus type II GRB optical afterglows," Astrophysical Journal Letters, vol. 734, no. 2, article 96, 2011.

[69] J. M. Cordes and T. J. W. Lazio, "NE2001.I. A new model for the galactic distribution of free electrons and its fluctuations," http://arxiv.org/abs/astro-ph/0207156.

[70] J. M. Cordes and T. J. W. Lazio, "NE2001. II. Using radio propagation data to construct a model for the galactic distribution of free electrons," http://arxiv.org/abs/astro-ph/ 0301598.

[71] H. Ardavan, A. Ardavan, and J. Singleton, "Frequency spectrum of focused broadband pulses of electromagnetic radiation generated by polarization currents with superluminally rotating distribution patterns," Journal of the Optical Society of America A, vol. 20, no. 11, pp. 2137-2155, 2003.

[72] H. Ardavan, A. Ardavan, J. Singleton, and M. R. Perez, "Mechanism of generation of the emission bands in the dynamic spectrum of the Crab pulsar," Monthly Notices of the Royal Astronomical Society, vol. 388, no. 2, pp. 873-883, 2008.

[73] H. Ardavan, A. Ardavan, J. Singleton et al., "Comparison of multiwavelength observations of 9 broad-band pulsars with the spectrum of the emission from an extended current with a superluminally rotating distribution pattern," http://arxiv.org/abs/0908.1349.

[74] A. A. Abdo, B. Allen, T. Aune et al., "The fermi gammaray space telescope discovers the pulsar in the young galactic supernova remnant CTA 1 (revised)," Science, vol. 322, pp. 1218-1221, 2008.

[75] W. S. Paciesas, C. A. Meegan, G. N. Pendleton et al., "The fourth BATSE gamma-ray burst catalog," Astrophysical Journal, Supplement Series, vol. 122, no. 2, pp. 465-495, 1999.

[76] H. Ardavan, P. Volegov, J. Middleditch et al., "The production mechanism of gamma-ray bursts,". In press.

[77] J. P. Norris and J. T. Bonnell, "Short gamma-ray bursts with extended emission," Astrophysical Journal, vol. 643, no. 1, pp. 266-275, 2006.

[78] E. Nakar and T. Piran, "Time-scales in long gamma-ray bursts," Monthly Notices of the Royal Astronomical Society, vol. 331, no. 1, pp. 40-44, 2002.

[79] J. L. Racusin, E. W. Liang, D. N. Burrows et al., "Jet breaks and energetics of swift gamma-ray burst X-ray afterglows," Astrophysical Journal Letters, vol. 698, no. 1, pp. 43-74, 2009.

[80] C. C. Thöne, A. de Ugarte Postigo, C. L. Fryer et al., "The unusual $\gamma$-ray burst GRB 101225A from a helium star/neutron star merger at redshift 0.33 ," Nature, vol. 480, pp. 72-74, 2011.

[81] S. Campana, G. Lodato, P. D’Avanzo et al., "The unusual gammaray burst GRB, 101225A explained as a minor body falling onto a neutron star," Nature, vol. 480, pp. 69-71, 2011.

[82] T. Siegfried, "Astronomy: surveys of exploding stars show one size does not fit all," Science, vol. 316, no. 5822, pp. 194-195, 2007.

[83] R. Di Stefano, "The progenitors of type Ia supernovae. II. Are they double-degenerate binaries? the symbiotic channel," Astrophysical Journal Letters, vol. 719, no. 1, pp. 474-482, 2010.

[84] R. A. Scalzo, G. Aldering, P. Antilogus et al., "Nearby supernova factory observations of SN 2007if: first total mass measurement of a super-chandrasekhar-mass progenitor," Astrophysical Journal Letters, vol. 713, no. 2, pp. 1073-1094, 2010.

[85] R. Pakmor, M. Kromer, S. Taubenberger, S. A. Sim, F. K. Röpke, and W. Hillebrandt, "Normal type Ia supernovae from violent mergers of white dwarf binaries," The Astrophysical Journal Letters, vol. 747, pp. L10-L14, 2012.

[86] B. E. Schaeffer and A. Pagnotta, "An absence of excompanion stars in the type Ia supernova remnant SNR, 0509-67. 5," Nature, vol. 481, pp. 164-166, 2012.

[87] J. S. Bloom, D. Kasen, K. J. Shen et al., "A compact degenerate primary-star progenitor of SN, 2011fe," The Astrophysical Journal, vol. 744, pp. L17-L21, 2012.

[88] H. Tananbaum and The Chandra Observing Team, "Cassiopeia A," International Astronomical Union Circular 7246, 1999.

[89] M. M. Phillips, "The absolute magnitudes of type Ia supernovae," Astrophysical Journal Letters, vol. 413, no. 2, pp. L105-L108, 1993.

[90] L. Wang, D. Baade, and F. Patat, "Spectropolarimetric diagnostics of thermonuclear supernova explosions," Science, vol. 315, no. 5809, pp. 212-214, 2007.

[91] A. M. Khokhlov, "Nucleosynthesis in delayed detonation models of Type Ia supernovae," Astronomy \& Astrophysics, vol. 245, pp. 114-128, 1991.

[92] T. Plewa, A. C. Calder, and D. Q. Lamb, “Type Ia supernova explosion: gravitationally confined detonation," Astrophysical Journal, vol. 612, no. 1, pp. L37-L40, 2004.

[93] P. A. Mazzali, K. S. Kawabata, K. Maeda et al., "Astronomy: an asymmetric energetic type Ic supernova viewed off-axis, and a link to gamma ray bursts," Science, vol. 308, no. 5726, pp. 1284-1287, 2005. 
[94] C. J. Conselice, P. M. Vreeswijk, A. S. Fruchter et al., "Gamma-ray burst-selected high-redshift galaxies: comparison to field galaxy populations to $\mathrm{z} \sim 3$," Astrophysical Journal, vol. 633, no. 1, pp. 29-40, 2005.

[95] J. Middleditch, "A white dwarf merger paradigm for supernovae and gamma-ray bursts," Astrophysical Journal, vol. 601, no. 2, pp. L167-L170, 2004.

[96] S. Benetti, E. Cappellaro, P. A. Mazzali et al., "The diversity of type Ia supernovae: evidence for systematics?" Astrophysical Journal Letters, vol. 623, no. 2, pp. 1011-1016, 2005.

[97] R. Sunyaev, A. Kaniovsky, V. Efremov et al., "Discovery of hard X-ray emission from supernova 1987A," Nature, vol. 330, no. 6145, pp. 227-229, 1987.

[98] S. M. Matz, G. H. Share, M. D. Leising et al., "Gamma-ray line emission from SN1987A," Nature, vol. 331, no. 6155, pp. 416-418, 1988.

[99] W. A. Mahoney, L. S. Varnell, A. S. Jacobson et al., "Gammaray observations of ${ }^{56} \mathrm{Co}$ in SN, 1987A," The Astrophysical Journal, vol. 334, pp. L81-L85, 1988.

[100] W. R. Cook, D. M. Palmer, T. A. Prince, S. M. Schindler, C. H. Starr, and E. C. Stone, "An imaging observation of SN, 1987A at gamma-ray energies," The Astrophysical Journal, vol. 334, pp. L87-L90, 1988.

[101] P. A. Pinto and R. G. Eastman, "The type Ia supernova widthluminosity relation,” New Astronomy, vol. 6, no. 5, pp. 307319, 2001.

[102] K. Krisciunas, P. M. Garnavich, P. Challis et al., "Hubble space telescope observations of nine high-redshift essence supernovae," Astronomical Journal, vol. 130, no. 6, pp. 2453 2472, 2005.

[103] N. Smith, W. Li, R. J. Foley et al., "SN 2006gy: discovery of the most luminous supernova ever recorded, powered by the death of an extremely massive star like $\eta$ carinae," Astrophysical Journal, vol. 666, no. 2, pp. 1116-1128, 2007.

[104] P. E. Nugent, Supernova 2007bi, CBET, 929, 1, 2007.

[105] S. E. Woosley, S. Blinnikov, and A. Heger, "Pulsational pair instability as an explanation for the most luminous supernovae," Nature, vol. 450, no. 7168, pp. 390-392, 2007.

[106] K. S. Kawabata, M. Tanaka, K. Maeda et al., "Extremely luminous supernova 2006gy at late phase: detection of optical emission from supernova," Astrophysical Journal Letters, vol. 697, no. 1, pp. 747-757, 2009.

[107] A. Gal-Yam, P. Mazzali, E. O. Ofek et al., "Supernova 2007bi as a pair-instability explosion," Nature, vol. 462, no. 7273, pp. 624-627, 2009.

[108] T. Moriya, N. Tominaga, M. Tanaka, K. Maeda, and K. Nomoto, "A core-collapse supernova model for the extremely luminous type Ic supernova 2007bi: an alternative to the pair-instability supernova model," Astrophysical Journal Letters, vol. 717, no. 2, pp. L83-L86, 2010.

[109] S. F. Portegies Zwart and E. P. J. Van Den Heuvel, “A runaway collision in a young star cluster as the origin of the brightest supernova," Nature, vol. 450, no. 7168, pp. 388-389, 2007.

[110] R. D. Bleach, R. C. Henry, G. Fritz et al., "Evidence for a compact source of soft X-rays in the Cygnus Loop," The Astrophysical Journal, vol. 197, pp. L13-L17, 1975.

[111] J. C. Weatherall, "Streaming instability in relativistically hot pulsar magnetospheres," Astrophysical Journal Letters, vol. 428, no. 1, pp. 261-266, 1994.

[112] D. Pesce, "Limits on the brightness of a compact object in the remnant Of SN, 1987a," in Proceedings of the American Astronomical Society, vol. 219, p. 239.13, 2012.
[113] A. A. Abdo, M. Ackermann, M. Ajello et al., "The first fermi large area telescope catalog of gamma-ray pulsars," The Astrophysical Journal Supplement Series, vol. 187, pp. 460494, 2010.

[114] J. Middleditch and J. A. Kristian, "A search for young, luminous optical pulsars in extragalactic supernova remnants," The Astrophysical Journal, vol. 279, pp. 157-161, 1984.

[115] Q. Z. Liu, J. Van Paradijs, and E. P. J. Van Den Heuvel, "A catalogue of low-mass x-ray binaries," Astronomy and Astrophysics, vol. 368, no. 3, pp. 1021-1054, 2001.

[116] M. Livio and G. T. Bath, "Fast coherent oscillations in variable X-ray sources and bursters," Astronomy \& Astrophysics, vol. 116, pp. 286-292, 1982.

[117] J. 't Zand, "List of 95 Galactic Type-I X-ray bursters," 2011, http://www.sron.nl/ jeanz/bursterlist.html.

[118] P. Kaaret, Z. Prieskorn, J. J. M. In 'T Zand et al., "Evidence of $1122 \mathrm{~Hz}$ X-ray burst oscillations from the neutron star X-ray transient XTE J1739-285," Astrophysical Journal, vol. 657, no. 2, pp. L97-L100, 2007.

[119] K. O. Mason, J. Middleditch, J. E. Nelson, and N. E. White, "An optical burst from the star identified with the X-ray source 2S1254-690," Nature, vol. 287, no. 5782, pp. 516-518, 1980.

[120] R. Wijnands, "Accreting millisecond X-ray pulsars: recent developments," Highlights of Astronomy, vol. 15, pp. 121-125, 2010.

[121] E. B. Fomalont, B. J. Geldzahler, and C. F. Bradshaw, "Scorpius X-1: the evolution and nature of the twin compact radio lobes," Astrophysical Journal Letters, vol. 558, no. 1, pp. 283-301, 2001.

[122] E. L. Wright, E. W. Gottlieb, and W. Liller, "Optical studies of UHURU sources. XII-the light curve of Scorpius X-1 = V818 Scorpii, 1889-1974," The Astrophysical Journal, vol. 200, pp. 171-176, 1975.

[123] I. J. Danziger and W. M. Goss, "The distance of Kepler's supernova remnant," Monthly Notices of the Royal Astronomical Society, vol. 190, pp. 47P-49P, 1980.

[124] S. van den Bergh, "The galactic supernovae of the second millenium, A. D.," Publications of the Astronomical Society of the Pacific, vol. 85, no. 505, pp. 335-340, 1973.

[125] K. A. Eriksen, D. Arnett, D. W. McCarthy, and P. Young, "The reddening toward cassiopeia A's supernova: constraining the ${ }^{56}$ NI yield," Astrophysical Journal Letters, vol. 697, no. 1, pp. 29-36, 2009.

[126] R. Rothenflug, J. Ballet, G. Dubner, E. Giacani, A. Decourchelle, and P. Ferrando, "Geometry of the nonthermal emission in SN 1006: azlmuthal variations of cosmic-ray acceleration," Astronomy and Astrophysics, vol. 425, no. 1, pp. 121-131, 2004.

[127] D. Milisavljevic and R. Fesen, "A kinematic map of the optical ejecta in the cas a supernova remnant," Bulletin of the American Astronomical Society, vol. 42, p. 469, 2010.

[128] A. Golden, A. Shearer, R. M. Redfern et al., "High speed phase-resolved 2-d UBV photometry of the Crab pulsar," Astronomy and Astrophysics, vol. 363, no. 2, pp. 617-628, 2000.

[129] C. Germanà, L. Zampieri, C. Barbieri et al., "Aqueye optical observations of the Crab Nebula pulsar," Astronomy and Astrophysics, vol. 548, pp. 47-53, 2012.

[130] T. H. Hankins and J. A. Eilek, "Radio emission signatures in the crab pulsar," Astrophysical Journal Letters, vol. 670, no. 1, pp. 693-701, 2007.

[131] R. P. Mignani, L. Pulone, G. Iannicola, G. G. Pavlov, L. Townsley, and O. Y. Kargaltsev, "Search for the elusive optical 
counterpart of PSR J0537-6910 with the HST Advanced Camera for Surveys," Astronomy and Astrophysics, vol. 431, no. 2, pp. 659-665, 2005.

[132] F. E. Marshall, E. V. Gorriielf, W. Zhang, J. Miooleditch, and Q. D. Wang, "Discovery of an ultrafast X-ray pulsar in the supernova remnant N157B," Astrophysical Journal Letters, vol. 499, no. 2, pp. L179-L182, 1998.

[133] K. M. Schure, J. Vink, A. Achterberg, and R. Keppens, "Evolution of magnetic fields and cosmic ray acceleration in supernova remnants," Advances in Space Research, vol. 44, no. 4, pp. 433-439, 2009.

[134] D. P. Finkbeiner, "Microwave interstellar medium emission observed by the Wilkinson Microwave Anisotropy Probe," Astrophysical Journal, vol. 614, no. 1, pp. 186-193, 2004.

[135] M. Su, T. R. Slatyer, and D. P. Finkbeiner, "Giant gamma-ray bubbles from Fermi-LAT: active Galactic nucleus activity or bipolar Galactic wind?” Astrophysical Journal Letters, vol. 724, no. 2, pp. 1044-1082, 2010.

[136] J. Chang, J. H. Adams, H. S. Ahn et al., "An excess of cosmic ray electrons at energies of 300-800 GeV," Nature, vol. 456, no. 7220, pp. 362-365, 2008.

[137] A. A. Abdo, M. Ackermann, M. Ajello et al., "Measurement of the cosmic ray $\mathrm{e}^{+}+\mathrm{e}^{-}$spectrum from $20 \mathrm{GeV}$ to $1 \mathrm{TeV}$ with the fermi Large Area Telescope," Physical Review Letters, vol. 102, no. 18, Article ID 1811016, 6 pages, 2009.

[138] O. Adriani, G. C. Barbarino, G. A. Bazilevskaya et al., "An anomalous positron abundance in cosmic rays with energies 1.5-100 GeV," Nature, vol. 458, no. 7238, pp. 607-609, 2009.

[139] M. R. Mumpower, G. C. McLaughlin, and R. Surman, "Formation of the rare earth peak: gaining insight into latetime r-process dynamics," Physical Review C, vol. 85, no. 4, Article ID 045801, 13 pages, 2011.

[140] B. Margon, H. C. Ford, J. I. Katz et al., "The bizarre spectrum of SS 433," The Astrophysical Journal, vol. 230, pp. 41L-45L, 1979.

[141] W. Kundt, “SS 433," MmSAI, vol. 70, pp. 1097-1102, 1999.

[142] S. N. Fabrika and L. V. Bychkova, "The mass function of SS 433," Astronomy \& Astrophysics, vol. 240, pp. L5-L7, 1990.

[143] T. Zwitter, S. D'Odorico S, T. Oosterloo, and M. Calvani, “On the masses and on the mass transfer in the interactive binary SS:433," in Proceedings of the International Astronomical Union Symposium (IAUS '92), vol. 151, pp. 465-467, 1992.

[144] K. M. Blundell, M. G. Bowler, and L. Schmidtobreick, "SS 433: observation of the circumbinary disk and extraction of the system mass," Astrophysical Journal Letters, vol. 678, no. 1, pp. L47-L50, 2008.

[145] K. Kubota, Y. Ueda, S. Fabrika et al., "Subaru and gemini observations of SS 433: new constraint on the mass of the compact object," The Astrophysical Journal, vol. 709, pp. 1374-1386, 2010.

[146] S. A. Grandi, "SS 433: how do the moving lines move?" Vistas in Astronomy, vol. 25, no. 1, pp. 7-11, 1981.

[147] B. Margon, "Observations of SS 433," Annual Review of Astronomy and Astrophysics, vol. 22, pp. 507-536, 1984.

[148] S. K. Chakrabarti, S. Pal, A. Nandi, B. G. Anandarao, and S. Mondal, "Possible photometric evidence of ejection of bullet-like features in the relativistic jet source SS 433," Astrophysical Journal Letters, vol. 595, no. 1, pp. L45-L48, 2003.

[149] J. C. Brown, J. P. Cassinelli, and G. W. Collins, "Constraints on the physical properties of optical bullets in SS 433," Astrophysical Journal Letters, vol. 378, no. 1, pp. 307-314, 1991.

[150] G. M. Beskin, S. I. Neizvestny, A. A. Pimonov, V. L. Plokhotnichenko, and V. F. Shvartsman, "A search for variability of the object SS4433 in the time range 10-61SECONDS according to 6-METER telescope observations," Astronomicheskii Tsirkulyar, no. 1087, p. 1B, 1979.

[151] E. Bertschinger, "Simulations of structure formation in the universe," Annual Review of Astronomy and Astrophysics, vol. 36, no. 1, pp. 599-654, 1998.

[152] T. A. Oosterloo and R. Morganti, "Anomalous H I kinematics in Centaurus A: evidence for jet-induced star formation," Astronomy and Astrophysics, vol. 429, no. 2, pp. 469-475, 2005.

[153] M. A. Dopita, M. Reuland, W. Van Breugel et al., "Halo ejection in distant radio galaxies: jet feedback in massive galaxy formation," Astrophysics and Space Science, vol. 311, no. 1-3, pp. 305-309, 2007.

[154] H. Tadhunter, R. A. E. Fosbury, and P. J. Quinn, "The kinematics of the ionized gas around powerful radio galaxies," Monthly Notices of the Royal Astronomical Society, vol. 240, pp. 225-254, 1989.

[155] W. van Breugel, C. Fragile, S. Croft, W. de Vries, P. Anninos, and S. Murray, "Jet-induced star formation: good news from big, bad black holes," Proceedings of the International Astronomical Union, vol. 222, pp. 485-488, 2004.

[156] O. 'Dowd M. J, D. Schiminovich, B. D. Johnson et al., "SSGSS: the spitzer-SDSS-GALEX spectroscopic survey," The Astrophysical Journal, vol. 741, pp. 79-118, 2011.

[157] W. Clavin, "NASA's Galaxy Evolution Explorer Helps Confirm Nature of Dark Energy," 2011, http://www.galex.caltech .edu/newsroom/glx2011-01r.html.

[158] K. Chen and M. Ruderman, "Origin and radio pulse properties of millisecond pulsars," Astrophysical Journal Letters, vol. 408, no. 1, pp. 179-185, 1993.

[159] M. Ruderman, "Spin-driven changes in neutron star magnetic fields," Journal of Astrophysics and Astronomy, vol. 16, no. 2, pp. 207-216, 1995.

[160] E. P. J. van den Heuvel, "High space velocities of single radio pulsars versus low orbital eccentricities and masses of double neutron stars: evidence for two different neutron star formation mechanisms," New Astronomy Reviews, vol. 54, no. 3-6, pp. 140-144, 2010.

[161] Y. Wang and M. Tegmark, "Uncorrelated measurements of the cosmic expansion history and dark energy from supernovae," Physical Review D, vol. 72, Article ID 103513, 7 pages, 2005.

[162] K. Heitmann, P. M. Ricker, M. S. Warren, and S. Habib, "Robustness of cosmological simulations. I. Large-scale structure," Astrophysical Journal, Supplement Series, vol. 160, no. 1 , pp. 28-58, 2005.

[163] U. Seljak and M. S. Warren, "Large-scale bias and stochasticity of haloes and dark matter," Monthly Notices of the Royal Astronomical Society, vol. 355, no. 1, pp. 129-136, 2004.

[164] E. Nelson and K. Petrillo, "An observational test of lambdaCDM galaxy formation," Bulletin of the American Astronomical Society, vol. 39, no. 1, p. 184, 2007.

[165] B. F. Madore, E. Nelson, and K. Petrillo, "Atlas and catalog of collisional ring galaxies," Astrophysical Journal, Supplement Series, vol. 181, no. 2, pp. 572-604, 2009.

[166] D. N. Spergel, L. Verde, H. V. Peiris et al., "First-year Wilkinson Microwave Anisotropy Probe (WMAP) observations: determination of cosmological parameters," Astrophysical Journal, Supplement Series, vol. 148, no. 1, pp. 175-194, 2003.

[167] K. S. Cheng, C. Ho, and M. Ruderman, "Energetic radiation from rapidly spinning pulsars. I-outer magnetosphere gaps. II-VELA and Crab," The Astrophysical Journal, vol. 300, pp. 500-539, 1986. 
[168] J. Pétri, "A unified polar cap/striped wind model for pulsed radio and gamma-ray emission in pulsars," Monthly Notices of the Royal Astronomical Society, vol. 412, no. 3, pp. 18701880, 2011.

[169] J. Pétri and G. Dubus, "Implication of the striped pulsar wind model for gamma-ray binaries," Monthly Notices of the Royal Astronomical Society, vol. 417, pp. 532-540, 2011.

[170] J. D. Jackson, Classical Electrodynamics, John Wiley \& Sons, 3rd edition, 1999.

[171] M. C. Weisskopf, R. F. Elsner, J. J. Kolodziejczak, S. L. O’Dell, and A. F. Tennant, "Unraveling the geometry of the crab nebula's "Inner Ring'", The Astrophysical Journal, vol. 746, pp. 41-43, 2012.

[172] S. Covino, D. Lazzati, G. Ghisellini et al., "GRB 990510: linearly polarized radiation from a fireball," Astronomy \& Astrophysics, vol. 348, pp. L1-L4, 1999.

[173] R. A. M. J. Wijers, P. M. Vreeswijk, T. J. Galama et al., "Detection of polarization in the afterglow of GRB 990510 with the ESO very large telescope," Astrophysical Journal Letters, vol. 523, no. 1, pp. L33-L36, 1999.

[174] E. Rol, R. A. M. J. Wijers, P. M. Vreeswijk et al., "GRB 990712: first indication of polarization variability in a gamma-ray burst afterglow," Astrophysical Journal, vol. 544, no. 2, pp. 707-711, 2000.

[175] D. Bersier, B. McLeod, P. M. Garnavich et al., "The strongly polarized afterglow of GRB 020405," Astrophysical Journal Letters, vol. 583, no. 2, pp. L63-L66, 2003.

[176] D. Götz, P. Laurent, F. Lebrun, F. Daigne, and E. Bonjak, "Variable polarization measured in the prompt emission of GRB 041219a using ibis on board integral," Astrophysical Journal Letters, vol. 695, no. 2, pp. L208-L212, 2009.

[177] M. F. Bietenholz, N. Bartel, and M. P. Rupen, "Discovery of a compact radio component in the center of supernova 1986J," Science, vol. 304, no. 5679, pp. 1947-1949, 2004.

[178] S. M. Ransom, J. W. T. Hessels, I. H. Stairs et al., "Twentyone millisecond pulsars in Terzan 5 using the Green Bank Telescope," Science, vol. 307, no. 5711, pp. 892-896, 2005.

[179] J. W. T. Hessels, S. M. Ransom, I. H. Stairs, P. C. C. Freire, V. M. Kaspi, and F. Camilo, "A radio pulsar spinning at $716 \mathrm{~Hz}$," Science, vol. 311, no. 5769, pp. 1901-1904, 2006.

[180] D. J. Champion, S. M. Ransom, P. Lazarus et al., "An eccentric binary millisecond pulsar in the galactic plane," Science, vol. 320, no. 5881, pp. 1309-1312, 2008.

[181] P. C. C. Freire, C. G. Bassa, N. Wex et al., "On the nature and evolution of the unique binary pulsar J1903+0327," Monthly Notices of the Royal Astronomical Society, vol. 412, no. 4, pp. 2763-2780, 2011.

[182] D. M. Palmer, S. Barthelmy, N. Gehrels et al., "A giant $\gamma$-ray flare from the magnetar SGR 1806-20," Nature, vol. 434, no. 7037, pp. 1107-1109, 2005.

[183] M. V. Lowson, "Focusing of helicopter BVI noise," Journal of Sound and Vibration, vol. 190, no. 3, pp. 477-494, 1996.

[184] J. M. Myers, H. M. Shen, T. T. Wu, and H. E. Brandt, "Curved electromagnetic missiles," Journal of Applied Physics, vol. 65, no. 7, pp. 2604-2610, 1989.

[185] T. Gold, “The 'Double Bang' of supersonic aircraft," Nature, vol. 170, no. 4332, p. 808, 1952.

[186] G. M. Lilley, R. Westley, A. H. Yates, and J. R. Busing, "The supersonic bang," Nature, vol. 171, no. 4362, pp. 994-996, 1953.

[187] T. A. Thompson, P. Chang, and E. Quataert, "Magnetar spindown, hyperenergetic supernovae, and gamma-ray bursts," Astrophysical Journal, vol. 611, no. 1, pp. 380-393, 2004.
[188] J. Vink and L. Kuiper, "Supernova remnant energetics and magnetars: no evidence in favour of millisecond protoneutron stars," Monthly Notices of the Royal Astronomical Society, vol. 370, no. 1, pp. L14-L18, 2006.

[189] T. Sakao, Y. Komura, K. Makishima et al., "When will the peculiar X-ray pulsar GX $1+4$, resume the fast spin-up?" Monthly Notices of the Royal Astronomical Society, vol. 246, pp. 11P-14P, 1990.

[190] J. Middleditch and J. E. Nelson, "Studies of optical pulsations from HZ Herculis/Hercules X-1: a determination of the mass of the neutron star," The Astrophysical Journal, vol. 208, pp. 567-586, 1976.

[191] J. Middleditch, "Optical pulsations from HZ Herculis/ Hercules X- 1-the self-consistent 35 day picture," The Astrophysical Journal, vol. 275, pp. 278-291, 1983.

[192] C. A. Jones, W. Forman, and W. Liller, "Optical studies of UHURU sources.IV. The long-term behavior of HZ HERCULIS=HERCULES X- 1," The Astrophysical Journal, vol. 182, pp. L109-L112, 1973.

[193] M. Kramer, I. H. Stairs, R. N. Manchester et al., "Tests of general relativity from timing the double pulsar," Science, vol. 314, no. 5796, pp. 97-102, 2006.

[194] L. Kasian, "Timing and precession of the young, relativistic binary pulsar PSR J1906+0746," in 40 Years of Pulsars: Millisecond Pulsars, Magnetars and More, C. Bassa, Z. Wang, A. Cumming, and V. M. Kaspi, Eds., AIP Conference Proceedings, pp. 485-487, August 2007.

[195] D. R. Lorimer, I. H. Stairs, P. C. Freire et al., "Arecibo pulsar survey using alfa. ii. the young, highly relativistic binary pulsar J1906+0746," Astrophysical Journal, vol. 640, no. 1, pp. 428-434, 2006.

[196] B. Kiziltan, A. Kotttas, and S. E. Thorsett, "The neutron star mass distribution," http://arxiv.org/abs/1011.4291. 

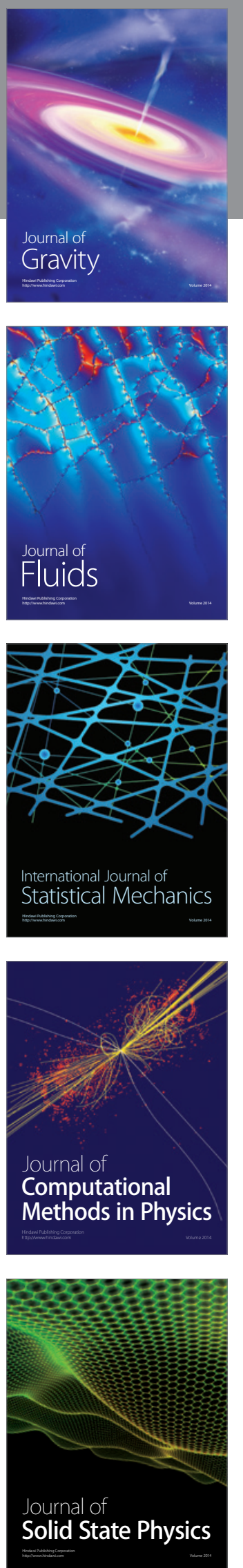

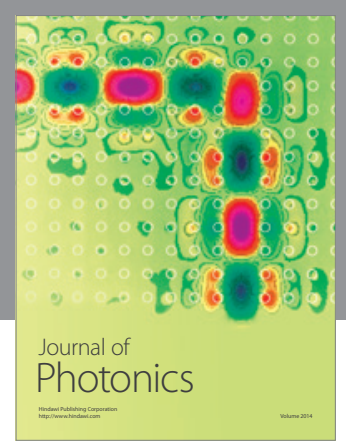

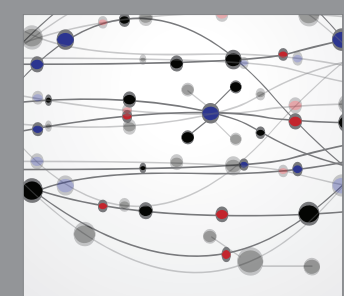

The Scientific World Journal
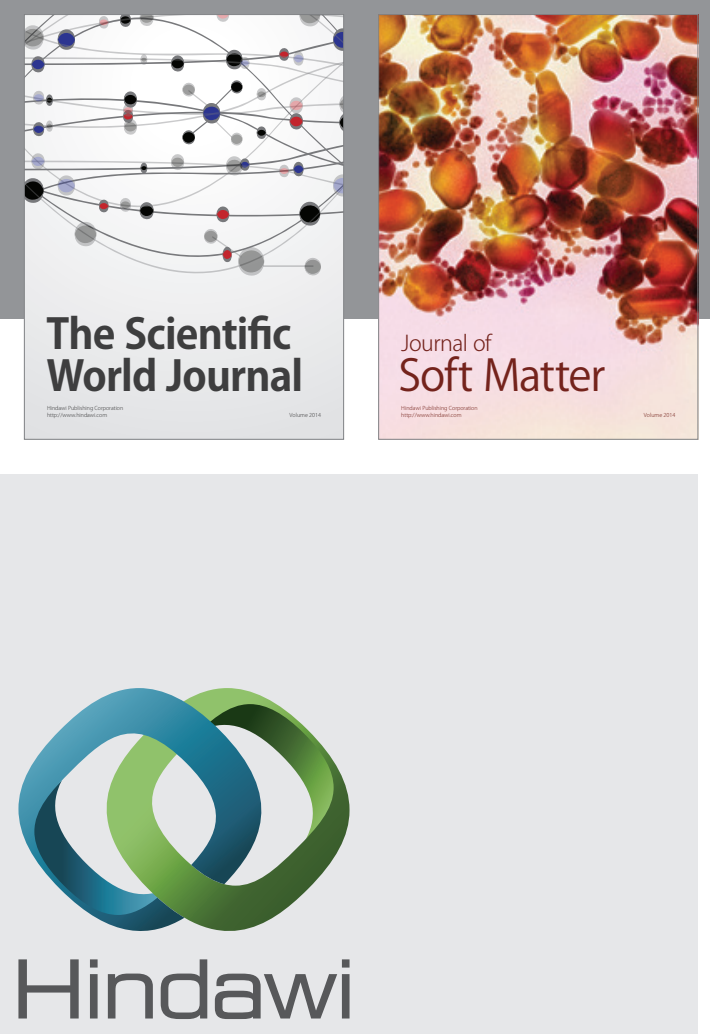

Submit your manuscripts at

http://www.hindawi.com
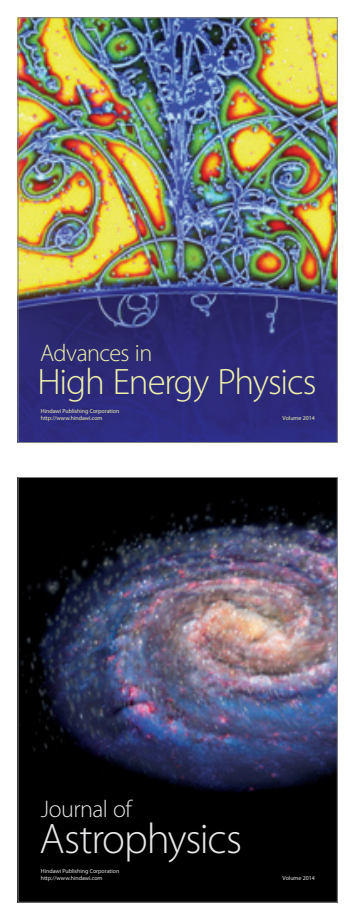
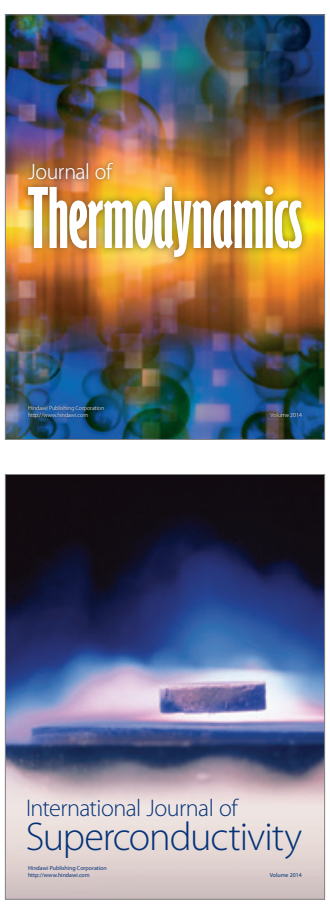
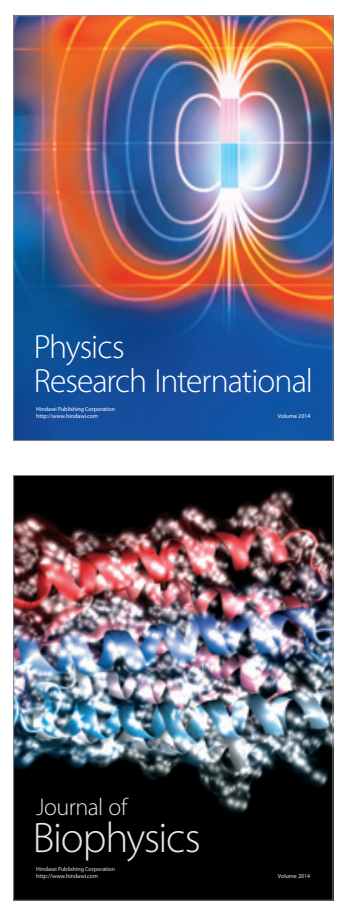
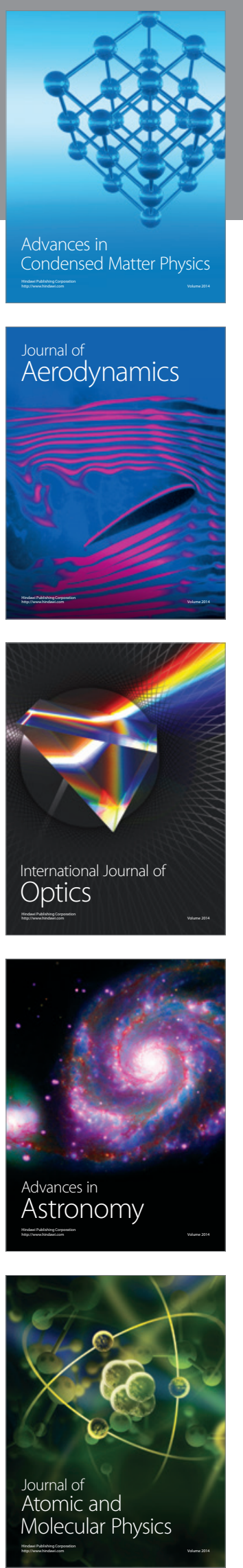